\title{
Condition de non-résonance pour l'oscillateur harmonique quantique perturbé
}

\author{
Rafik Imekraz
}

Communicated by Yvan Martel, received November 21, 2011.

\begin{abstract}
On $\mathbb{R}^{d}$, we consider the differential operator $-\Delta+\|x\|^{2}+R(x)+M$. Here $R$ is in the Schwartz class and $M$ is a bounded operator of $L^{2}(\mathbb{R})$. Under a localization hypothesis on the spectrum of $-\Delta+\|x\|^{2}+R(x)$, which is automatically satisfied if $d=1$, one proves that there are arbitrarily small operator $M$, i.e. $\|M\| \ll 1$, such that for all $r \geq 3$, for small Cauchy data of order $\varepsilon$ in high Sobolev norms, the nonlinear Schrödinger equation admits a solution which is bounded by $2 \varepsilon$ on a time existence interval of length $\geq \varepsilon^{-r}$. The case $R=0$ has been proved by Grébert-Imekraz-Paturel. Here we do not need any explicit spectral analysis (eigenvalues and eigenfunctions) of $-\Delta+$ $\|x\|^{2}+R(x)$. The role of $M$ is making the spectrum of $-\Delta+\|x\|^{2}+R(x)+M$ nonresonant. We also give a partial answer in the case $M=0$ : there are some potentials $R$ such that the spectrum of $-\partial_{x}^{2}+x^{2}+R(x)$ is nonresonant. For this, we use some Chelkak-Kargaev-Korotyaev's results about the inverse spectral problem of harmonic oscillator and construct explicit dual basis of the squared Hermite functions.

Sur $\mathbb{R}^{d}$, nous considérons l'opérateur différentiel $-\Delta+\|x\|^{2}+R(x)+$ $M$ où $R$ appartient à la classe de Schwartz et $M$ est un opérateur borné de $L^{2}(\mathbb{R})$. Sous une hypothèse de localisation du spectre de $-\Delta+\|x\|^{2}+R(x)$, automatiquement satisfaite si $d=1$, nous prouvons l'existence d'opérateurs $M$ arbitrairement petits, i.e. $\|M\| \ll 1$, tels que pour tout $r \geq 3$ et pour toute donnée initiale d'ordre $\varepsilon$ dans un espace de Sobolev à grande régularité, l'équation de Schrödinger non-linéaire admet une solution bornée par $2 \varepsilon$ sur un intervalle temporel de longueur $\geq \varepsilon^{-r}$. Le cas $R=0$ a été démontré par Grébert-Imekraz-Paturel. Ici, nous n'avons pas besoin de connaître les valeurs exactes des valeurs propres et des modes propres de $-\Delta+\|x\|^{2}+R(x)$. Le rôle de $M$ est de rendre non-résonant le spectre de $-\Delta+\|x\|^{2}+R(x)+M$. Nous donnons aussi une réponse partielle dans le cas $M=0$ : il existe des potentiels $R$ tels que le spectre de $-\partial_{x}^{2}+x^{2}+R(x)$ est non-résonant. Pour cela, nous invoquons des résultats de Chelkak-Kargaev-Korotyaev concernant le problème inverse spectral de l'oscillateur harmonique et construisons une base duale des carrées des fonctions de Hermite.
\end{abstract}

1991 Mathematics Subject Classification. 35.

Key words and phrases. Differential operator, spectrum, nonlinear Schrödinger equation. 
TABLE DES MATIÈRES

1. Introduction 206

2. Énoncé précis des théorèmes 208

3. Analyse spectrale 211

4. Modèle hamiltonien 218

5. Potentiels non-résonants en dimension 1 et base duale 223

$\begin{array}{ll}\text { References } & 237\end{array}$

\section{Introduction}

Nous nous intéressons au comportement dynamique des solutions de l'équation de Schrödinger dans les espaces de Sobolev à grande régularité $s \gg 1$

(1) $\begin{cases}i \partial_{t} \psi & =\left(-\Delta+\|x\|^{2}+R(x)+M\right) \psi \pm|\psi|^{2 p} \psi \quad(t, x) \in \mathbb{R}^{d} \times \mathbb{R} \\ \psi_{0} & \in \widehat{H}^{s}\end{cases}$

Nous généralisons les résultats de [GIP09] (cas $R=0)$. Nous notons

- $R$ appartient à l'espace $\mathcal{B C}^{\infty}\left(\mathbb{R}^{d}, \mathbb{R}\right)$ des fonctions de classe $\mathcal{C}^{\infty}$, bornées et à dérivées bornées.

- Nous posons $T:=-\Delta+\|x\|^{2}+R(x)$. Les espaces de Sobolev $\widehat{H}^{s}:=\operatorname{Dom}\left(T^{s / 2}\right)=$ $\left\{f \in H^{s}\left(\mathbb{R}^{d}\right),\langle x\rangle^{s} f(x) \in L^{2}\left(\mathbb{R}^{d}\right)\right\}$ de $T$ sont munis des normes naturelles $\|\cdot\|_{\widehat{H}^{s}}$.

- $M$ appartient à l'espace $\operatorname{Com}(T)$ des opérateurs compacts de $L^{2}\left(\mathbb{R}^{d}\right)$ qui commutent avec $T$ et l'on note

$$
\forall M \in \operatorname{Com}(T) \quad\|M\|=\sup _{f \neq 0} \frac{\|M f\|_{L^{2}\left(\mathbb{R}^{d}\right)}}{\|f\|_{L^{2}\left(\mathbb{R}^{d}\right)}}
$$

Dans la suite, on aura $\|M\| \ll 1$.

Notre premier théorème repose sur une hypothèse de localisation du spectre de $T=-\Delta+\|x\|^{2}+R(x)$ que nous préciserons plus loin mais qui implique que l'on a

(2) $\exists \sigma, c \geq 0 \quad \exists \delta>0 \quad \operatorname{sp}(T) \subset \bigcup_{j \geq 1} K_{j}, \quad K_{j}:=\left[2 j+\sigma-\frac{c}{j^{\delta}}, 2 j+\sigma+\frac{c}{j^{\delta}}\right]$

Notre hypothèse sera en fait presque automatique en dimension 1 . Notre premier résultat d'existence presque globale dans les espaces de Sobolev à grande régularité s'énonce

Théorème 1.1. Il existe des opérateurs compacts $M: L^{2}\left(\mathbb{R}^{d}\right) \rightarrow \mathbb{R}$ qui commutent avec $T$ et de norme $\|M\| \ll 1$ tels que pour tous entiers $r \geq 3$ et $s \gg 1$, si $\varepsilon:=\left\|\psi_{0}\right\|_{\widehat{H}^{s}} \ll 1$ alors l'équation de Schrödinger perturbée :

$$
i \partial_{t} \psi=\left(-\Delta+\|x\|^{2}+R(x)+M\right) \psi \pm|\psi|^{2 p} \psi
$$

admet une unique solution $\psi(t, x)$ dans l'espace $\mathcal{C}^{0}\left(\left(-C \varepsilon^{-r}, C \varepsilon^{-r}\right), \widehat{H}^{s}\right)$. En outre, on a le contrôle

$$
|t| \leq C \varepsilon^{-r} \quad \Rightarrow \quad\|\psi(t, \cdot)\|_{\widehat{H}^{s}} \leq 2 \varepsilon
$$


Le théorème 1.1 a été prouvé dans le cas particulier $R=0$ dans [GIP09]. Dans le cas $R=0$, le spectre de $-\Delta+\|x\|^{2}$ est $d+2 \mathbb{N}$ et les fonctions propres sont les fonctions de Hermite. Nous améliorons la méthode de [GIP09] pour obtenir l'existence presque globale dans le cas $R \neq 0$ quand le spectre et les valeurs propres ne sont pas connus.

Dans le théorème 1.1, le but de l'opérateur $M$ est de faire en sorte que l'opérateur différentiel $-\partial_{x}^{2}+x^{2}+R(x)+M$ a un spectre non-résonant, c'est-à-dire que les combinaisons linéaires de ses valeurs propres ne s'approchent pas trop près de zéro en un sens que nous définirons plus loin. Notre second théorème donne une réponse partielle au cas $M=0$ en une dimension.

Théorème 1.2. Soit $\left(h_{n}\right)_{n \geq 0}$ la base hilbertienne de Hermite de $L^{2}(\mathbb{R})$. Il existe une unique famille $\left(h_{n}^{\star}\right)_{n \geq 0}$ de fonctions paires de $L^{2}(\mathbb{R})$ telle que

$$
\forall(n, m) \in \mathbb{N}^{2} \quad \int_{\mathbb{R}} h_{n}^{2}(x) h_{m}^{\star}(x)=\delta_{n, m}
$$

De plus, pour des paramètres génériques $r_{n}$, le potentiel $R:=\sum_{n \geq 0} r_{n} h_{n}^{\star}$ appartient $\grave{a} \widehat{H}^{1}$ et le spectre de $-\partial_{x}^{2}+x^{2}+R(x)$ est non-résonant.

Les preuves des théorèmes 1.1 et 1.2 sont indépendantes.

Expliquons les grandes lignes de la preuve du théorème 1.1. On interprète l'équation (3) comme une équation hamiltonienne avec un hamiltonien $H_{0}+P$ sur un espace symplectique adapté. L'existence presque globale découle d'une procédure de formes normales, c'est-à-dire que l'on peut transformer le hamiltonien $H_{0}+P$ en un hamiltonien plus simple. Cette réduction est obtenue grâce aux estimations (4) qui permettent d'appliquer un théorème de formes normales présent dans [GIP09]. Nous ne referons pas la démonstration de ce dernier mais il nous paraît important d'expliquer que pour réduire le hamiltonien $H_{0}+P$, nous devons contrôler les combinaisons linéaires des valeurs propres de $-\Delta+\|x\|^{2}+R(x)$. Il s'agit du problème classique des petits diviseurs. C'est alors qu'intervient l'opérateur perturbatif $M$ dans l'équation (3), il a vocation à éviter les résonances des fréquences du hamiltonien libre. Contrairement à la preuve de [GIP09], l'opérateur $M$ est construit grâce à une argumentation de Delort et Szeftel [DS06b]. En effet, Delort et Szeftel ont montré dans [DS06b] que l'opérateur $\sqrt{-\Delta+m^{2}}$ sur une variété de Zoll évite les résonances pour presque tout $m>0$ grâce à une inclusion analogue à (2). Comme dans [GIP09], nous avons besoin des estimations suivantes des intégralesproduits des modes propres :

$$
\begin{gathered}
\exists \beta_{k}, \nu_{k}>0 \quad \forall N \geq 1 \quad \forall j_{1} \geq \cdots \geq j_{k} \quad \forall\left(\ell_{1}, \cdots \ell_{k}\right) \in \prod_{i=1}^{k}\left[\left[1, d_{i}\right]\right] \\
\left|\int_{\mathbb{R}^{d}} \phi_{j_{1}}(x) \cdots \phi_{j_{k}}(x) d x\right| \leq C_{N} \frac{\lambda_{j_{3}}^{\nu}}{\lambda_{j_{1}}^{\beta}}\left(\frac{\sqrt{\lambda_{j_{2}} \lambda_{j_{3}}}}{\sqrt{\lambda_{j_{2}} \lambda_{j_{3}}}+\lambda_{j_{1}}-\lambda_{j_{2}}}\right)^{N}
\end{gathered}
$$

La preuve de ces inégalités est facilitée dans le cas $R=0$ par la donnée exacte des modes propres et du spectre. En particulier, les modes propres de $-\Delta+\|x\|^{2}$ sont obtenus par produit tensoriel des fonctions de Hermite uni-dimensionnelles et l'on peut par conséquent vérifier que $\left\|\phi_{\lambda}\right\|_{L^{\infty}} \leq C \lambda^{-1 / 12}$. Dans le cas $R \neq 0$, nous ignorons si cette estimation est encore valable. Néanmoins, grâce aux estimations des normes $L^{p}\left(\mathbb{R}^{d}\right)$ des modes propres obtenues dans [KT05](ces dernières ont des 
formes différentes selon que $d=1$ ou $d \geq 2$, voir le théorème 3.9 du présent article), nous arrivons à prouver les estimations (4).

Expliquons à présent formellement la preuve du théorème 1.2. À $n$ et $R$ fixés, la fonction $\lambda_{n}$ satisfait le développement limité :

$$
\forall \varepsilon \in]-1,1\left[\quad \lambda_{n}(\varepsilon R)=2 n+1+\varepsilon \int_{\mathbb{R}} h_{n}^{2}(x) R(x) d x+o(\varepsilon)\right.
$$

Grâce à l'existence d'une série génératrice pour les fonctions de Hermite, nous allons prouver qu'il existe des fonctions $h_{n}^{\star} \in \widehat{H}^{1}(\mathbb{R})$ telles que

$$
\forall(n, m) \in \mathbb{N}^{2} \quad \int_{\mathbb{R}} h_{m}^{\star}(x) h_{n}^{2}(x) d x=\delta_{n, m}
$$

Par suite, pour tout $R=\sum_{n \geq 0} r_{n} h_{n}^{\star}$, le développement limité (5) devient

$$
\lambda_{n}(\varepsilon R)=2 n+1+\varepsilon r_{n}+o(\varepsilon)
$$

Heuristiquement, cela signifie que l'on peut perturber de manière indépendante chaque valeur propre $\lambda_{n}(\varepsilon R)$ grâce aux paramètres indépendants $r_{n}$ et cela va nous donner assez de liberté pour obtenir des potentiels non-résonants. Nous allons mettre en place cette idée rigoureusement grâce à la théorie du problème spectral inverse de Chelkak-Kargaev-Korotyaev [CKK04]. L'idée de considérer la base duale finie de $\left(h_{n}^{2}\right)_{0 \leq n<N}$ a déjà été utilisée par Grébert et Thomann pour perturber un nombre fini $N$ de valeurs propres dans [GT11, lemme 6.3]. L'existence de la base duale est évidente dans le cas fini. Notre cas est plus compliqué car nous voulons perturber un nombre infini de valeurs propres et l'existence d'une base duale est fausse en général (voir la remarque 5.4).

Permettons-nous à présent de mentionner quelques travaux antérieurs concernant des résultats d'existence presque globale analogues au théorème 1.1 obtenus par des méthodes de formes normales. La technique utilisée à été développée par Bambusi [Bam08], Bambusi-Grébert ([BG04, BG06]) et Faou-Grébert [FG10] pour des EDP sur le tore, Delort-Szeftel pour l'équation semi-linéaire de KleinGordon sur des surfaces de révolution compactes [DS06a], les sphères [DS04] et les variétés de Zoll [DS06b]. Rappelons qu'une variété de Zoll est une variété riemannienne compacte dont le flot géodésique est simplement périodique [Bes78], par exemple une sphère ou un espace projectif. À notre connaissance, l'un des cas les plus généraux traités est l'équation semi-linéaire de Klein-Gordon sur les variétés de Zoll avec des formes normales à tout ordre par Bambusi-Delort-GrébertSzeftel [BDGS07]. En ce qui concerne les variétés non compactes, hormis l'article [GIP09], l'oscillateur anharmonique $-\partial_{x}^{2}+x^{2 p}$ sur $\mathbb{R}$ a été traité dans [Ime12] pour $p \geq 2$.

L'auteur remercie Patrick Bernard, Ivar Ekeland et Eric Seré de l'avoir conseillé sur les équations hamiltoniennes. L'auteur remercie Yves Colin de Verdière et Didier Robert pour les discussions spectrales. L'auteur remercie également JeanMarc Delort et Benoît Grébert de lui avoir suggéré des améliorations substantielles du présent texte.

\section{2. Énoncé précis des théorèmes}

Considérons une suite d'entiers non nuls $\left(d_{j}\right)_{j \geq 1}$ qui est à croissance polynomiale, i.e. $d_{j} \leq C j^{c}$. Nous allons indexer les valeurs propres à l'aide de l'ensemble 
$E^{+}$muni de l'ordre lexicographique :

$$
\begin{gathered}
E^{+}=\left\{(j, \ell) \in \mathbb{N}^{\star} \times \mathbb{N}^{\star}, \quad 1 \leq \ell \leq d_{j}\right\} \\
(j, 1)<\cdots<\left(j, d_{j}\right)<(j+1,1)<\cdots<\left(j+1, d_{j+1}\right)
\end{gathered}
$$

Définition 2.1. Soit $R \in \mathcal{B C}^{\infty}\left(\mathbb{R}^{d}, \mathbb{R}\right)$, nous dirons que le spectre de $T=$ $-\Delta+\|x\|^{2}+R(x)$ est Zoll-localisé, ou encore que la propriété $(Z)$ est vérifiée, s'il existe une suite $\left(d_{j}\right)_{j \geq 0}$ de sorte qu'en numérotant $S p(T)=\left(\lambda_{j, \ell}\right)_{E^{+}}$nous avons

$$
\exists \sigma, c \geq 0 \quad \exists \delta>0 \quad \forall(j, \ell) \in E^{+} \quad\left|\lambda_{j, \ell}-2 j-\sigma\right| \leq \frac{c}{j^{\delta}}
$$

La définition précédente implique l'inclusion suivante

$$
\mathrm{Sp}(T) \subset \bigcup_{j \geq 1} K_{j}, \quad K_{j}=\left[2 j+\sigma-\frac{c}{j^{\delta}}, 2 j+\sigma+\frac{c}{j^{\delta}}\right]
$$

Néanmoins la définition (2.1) est beaucoup plus précise et assure que la somme des multiplicités des valeurs propres de $T$ dans $K_{j}$ est $d_{j} \leq C j^{c}$. Voici quelques exemples pour lesquels la propriété $(\mathrm{Z})$ est vérifiée :

i) $R=0$, i.e. pour l'oscillateur harmonique $-\Delta+\|x\|^{2}$

ii) McKean et Trubowitz ont montré dans [MT82] le résultat de non isospectralité suivant : il existe toute une famille de potentiels $R \in \mathcal{S}(\mathbb{R})$ tels que le spectre de $-\partial_{x}^{2}+x^{2}+R(x)$ est exactement $1+2 \mathbb{N}$

iii) en dimension 1 un théorème de [KKP05] implique que si $R \in \mathcal{B C} C^{\infty}(\mathbb{R}) \cap L^{1}(\mathbb{R})$ alors $-\partial_{x}^{2}+x^{2}+R(x)$ vérifie automatiquement $(\mathrm{Z})$

Nous prouvons le théorème suivant d'existence presque globale dans les espaces de Sobolev à grande régularité :

Théorème 2.2. On suppose que

i) $-\Delta+\|x\|^{2}+R(x)$ vérifie $(Z)$

ii) $g: \mathbb{C}^{2} \rightarrow \mathbb{C}$ est une fonction holomorphe qui s'annule en $(0,0)$ avec multiplicité $\geq 3$, et $g(\xi, \bar{\xi}) \in \mathbb{R}$ pour tout $\xi \in \mathbb{C}$

Il existe une fonction analytique réelle $M:[0,1] \rightarrow \operatorname{Com}(T)$ vérifiant $\|M(m)\|<$ $C m$ et telle que pour presque tout $m \in] 0,1[$, pour tous $r \geq 3$ et $s \gg 1$, si $\varepsilon:=$ $\left\|\psi_{0}\right\|_{\widehat{H}^{s}} \ll 1$ alors l'équation de Schrödinger

$$
i \partial_{t} \psi=\left(-\Delta+\|x\|^{2}+R(x)+M(m)\right) \psi+\partial_{2} g(\psi, \bar{\psi})
$$

admet une unique solution $\psi(t, x)$ dans l'espace $\mathcal{C}^{0}\left(\left(-C \varepsilon^{-r}, C \varepsilon^{-r}\right), \widehat{H}^{s}\right)$. En outre, nous avons le contrôle

$$
|t|<C \varepsilon^{-r} \Rightarrow\|\psi(t, \cdot)\|_{\widehat{H}^{s}} \leq 2 \varepsilon
$$

et en décomposant $\psi(t, x)=\sum_{j \geq 1} \sum_{l=1}^{d_{j}} z_{j, l}(t) \phi_{j, l}(x)$ nous avons

$$
|t|<\left.C \varepsilon^{-r} \Rightarrow \sum_{j \geq 1} j^{s}\left|\sum_{l=1}^{d_{j}}\right| z_{j, l}(t)\right|^{2}-\left|z_{j, l}(0)\right|^{2} \mid \leq C \varepsilon^{3}
$$


Comme nous l'avons annoncé dans l'introduction, le cas $M=0$ semble intimement lié à la base duale carrée des fonctions de Hermite. Rappelons que la base hilbertienne de Hermite est définie par

$$
\forall x \in \mathbb{R} \quad h_{n}(x)=(-1)^{n} \frac{e^{x^{2} / 2} \partial_{x}^{n}\left(e^{-x^{2}}\right)}{\pi^{1 / 4} \sqrt{n ! 2^{n}}}, \quad\left(-\partial_{x}^{2}+x^{2}\right) h_{n}=(2 n+1) h_{n}
$$

On note $L_{\text {pair }}^{2}(\mathbb{R})=\left\{f \in L^{2}(\mathbb{R}), f(x)=f(-x) \quad\right.$ p.p. $\}$. Le théorème suivant résume les propriétés de la base duale carrée :

Théorème 2.3. Il existe une unique famille $\left(h_{n}^{\star}\right)_{n \geq 0}$ de $L_{\text {pair }}^{2}(\mathbb{R})$ telle que

$$
\forall(n, m) \in \mathbb{N}^{2} \quad \int_{\mathbb{R}} h_{m}^{\star}(x) h_{n}^{2}(x) d x=\delta_{n, m}
$$

En outre, cette famille vérifie les propriétés suivantes

i) on pose $\alpha_{n}=\frac{(2 n) !}{n !^{2} 4^{n}}$, les coordonnées de $h_{n}$ sur la base hilbertienne

$$
\left(h_{2 k}(\cdot \sqrt{2}) 2^{1 / 4}\right)_{k \geq 0}
$$

de $L_{\text {pair }}^{2}(\mathbb{R})$ sont données par

$$
h_{n}^{\star}(x)=\frac{(2 \pi)^{1 / 4}}{\sqrt{\alpha_{n}}} h_{2 n}(x \sqrt{2}) 2^{1 / 4}-\sum_{k=n+1}^{\infty}\left[\frac{(2 \pi)^{1 / 4} \alpha_{k-n}}{(2(k-n)-1) \sqrt{\alpha_{k}}}\right] h_{2 k}(x \sqrt{2}) 2^{1 / 4}
$$

ii) $h_{n}^{\star}$ appartient $\grave{a} \widehat{H}^{s}(\mathbb{R})$ si et seulement si $s<\frac{3}{2}$. La fonction $h_{n}^{\star}$ est donc continue, bornée et vérifie $\left\|h_{n}^{\star}\right\|_{L^{\infty}} \leq C n^{1 / 6}$.

En application du théorème précédent et de la théorie de Chelkak-KargaevKorotyaev [CKK04], nous montrons que génériquement un potentiel $R$ dans $\widehat{H}^{1}$ a un spectre non-résonant.

Théorème 2.4. Pour des paramètres génériques $\left(r_{n}\right)_{n}$, le potentiel $R:=\sum r_{n} h_{n}^{\star}$ appartient $\grave{a} \widehat{H}^{1}$ et le spectre $\left(\lambda_{n}\right)_{n \geq 0}$ de $-\partial_{x}^{2}+x^{2}+R(x)$ est non-résonant au sens suivant

$$
\begin{gathered}
\forall r \geq 3 \quad \forall k \in[[1, r-1]] \quad \exists \delta, \gamma>0 \quad \forall n_{1}, \cdots, n_{r} \in \mathbb{N} \\
\left\{n_{1}, \cdots, n_{k}\right\} \neq\left\{n_{k+1}, \cdots, n_{r}\right\} \Rightarrow\left|\sum_{i=1}^{k} \lambda_{n_{i}}-\sum_{i=k+1}^{r} \lambda_{n_{i}}\right| \geq \frac{\gamma}{\left(1+n_{3}^{\star}\right)^{\delta}}
\end{gathered}
$$

où $n_{3}^{\star}$ est le troisième plus grand entier parmi $n_{1}, \ldots, n_{r}$.

La définition précédente de non-résonance fait intervenir un nombre infini de valeurs propres, elle apparaît notamment dans [GIP09, Ime12, DS04, DS06b, BDGS07] et dans une version un peu différente dans [BG06].

Ce travail est divisé de la manière suivante : dans la partie 3 , nous expliquons toute l'analyse spectrale de $-\Delta+\|x\|^{2}+R(x)$ dont nous avons besoin. Dans la partie 4, nous mettons l'équation de Schrödinger sous forme hamiltonienne pour pouvoir appliquer un théorème de formes normales, cela montrera le théorème 2.2. La partie 5 est dévolue à la démonstration des théorèmes 2.3 et 2.4 . 


\section{Analyse spectrale}

3.1. Domaine spectral. Nous allons rappeler quelques résultats bien connus. La proposition suivante est importante et décrit les espaces de Sobolev de l'oscillateur harmonique (voir [Hel84]) :

Proposition 3.1. Définissons $T_{0}=-\Delta+\|x\|^{2}$. Pour tout $s \in \mathbb{R}$, le domaine de l'opérateur $T_{0}^{s / 2}$ est

$$
\widehat{H}^{s}\left(\mathbb{R}^{d}\right)=\left\{f \in L^{2}\left(\mathbb{R}^{d}\right), \quad\|f\|_{\widehat{H}^{s}}:=\|f\|_{H^{s}}+\left\|\langle x\rangle^{s} f(x)\right\|_{L^{2}}<+\infty\right\}
$$

On notera $\widehat{H}^{s}=\widehat{H}^{s}\left(\mathbb{R}^{d}\right)$ s'il n'y a pas ambiguïté. En outre, pour tout $s^{\prime} \in \mathbb{R}, T_{0}^{s}$ envoie continûment $\widehat{H}^{2 s^{\prime}}$ dans $\widehat{H}^{2\left(s^{\prime}-s\right)}$.

La chaîne d'espaces de Sobolev $\left(\widehat{H}^{s}\left(\mathbb{R}^{d}\right)\right)_{s \in \mathbb{R}}$ est munie de la dualité naturelle par rapport à $L^{2}\left(\mathbb{R}^{d}\right)$, si bien que le dual de $\widehat{H}^{s}\left(\mathbb{R}^{d}\right)$ s'identifie à $\widehat{H}^{-s}\left(\mathbb{R}^{d}\right)$. Nous avons aussi la

Proposition 3.2. Soit $R: \mathcal{S}\left(\mathbb{R}^{d}\right) \rightarrow \mathcal{S}\left(\mathbb{R}^{d}\right)$ un opérateur linéaire auto-adjoint qui conserve continûment tout espace $\widehat{H}^{s}$ pour $s \in \mathbb{R}$. Alors les modes propres de $T_{0}+R$ sont dans l'espace de Schwartz $\mathcal{S}\left(\mathbb{R}^{d}\right)$ et pour tout $s>0$ on a Dom $\left(T_{0}+R\right)^{s}=$ $\widehat{H}^{s}$.

Preuve. La régularité des modes propres découle d'un argument complètement standard : si $f \in L^{2}\left(\mathbb{R}^{d}\right)$ vérifie $T_{0} f=(\lambda-R) f$ alors $T_{0} f \in L^{2}\left(\mathbb{R}^{d}\right)$, donc $f \in \widehat{H}^{2}$. L'équation $T_{0} f=(\lambda-R) f \in \widehat{H}^{2}\left(\mathbb{R}^{d}\right)$ permet de nouveau de gagner de la régularité et une récurrence immédiate prouve que $f$ appartient à l'espace $\cap_{n \geq 0} \widehat{H}^{2 n}\left(\mathbb{R}^{d}\right)$ qui n'est autre que $\mathcal{S}\left(\mathbb{R}^{d}\right)$ (cela se voit par exemple à l'aide de la description des espaces de Sobolev donnée par la proposition 3.1).

Montrons maintenant l'égalité des domaines dans le cas où $s \in \mathbb{N}$. En développant $\left(T_{0}+R\right)^{s}$, l'opérateur différentiel $\left(T_{0}+R\right)^{s}-T_{0}^{s}$ apparaît comme une somme d'opérateurs différentiels $A_{1}, \cdots, A_{n}$ qui sont composés de $T_{0}$ et de $R$. Disons que $A_{i}$ est composé de $k_{i}$ fois $T_{0}$ et $s-k_{i}$ fois $R$ pour $k_{i} \in[0, s-1]$. Notons que la formule du binôme n'est pas applicable car $R$ et $T_{0}$ ne commutent pas a priori. L'opérateur $A_{i} \circ T_{0}^{1-s}$ envoie continûment $L^{2}=\widehat{H}^{0}$ dans $\widehat{H}^{2\left(s-1-k_{i}\right)} \subset L^{2}$. Cela se traduit par

$$
\forall f \in \mathcal{S}\left(\mathbb{R}^{d}\right) \quad\left\|\left(T_{0}+R\right)^{s} f-T_{0}^{s} f\right\|_{L^{2}\left(\mathbb{R}^{d}\right)} \leq C\left\|T_{0}^{s-1} f\right\|_{L^{2}\left(\mathbb{R}^{d}\right)}
$$

A présent, nous allons faire intervenir la base hilbertienne de Hermite multidimensionnelle $\left(h_{j, \ell}\right)$ avec $T_{0} h_{j, \ell}=(2(j-1)+d) h_{j, \ell}$ et $1 \leq \ell \leq C j^{d-1}$. Ainsi,

$$
\frac{1}{C} \sum_{(j, \ell)} j^{2(s-1)}\left\langle f, h_{j, \ell}\right\rangle^{2} \leq\left\|T_{0}^{s-1} f\right\|_{L^{2}\left(\mathbb{R}^{d}\right)}^{2} \leq C \sum_{(j, \ell)} j^{2(s-1)}\left\langle f, h_{j, \ell}\right\rangle^{2}
$$

Or pour tout $a>0$ il existe $b>0$ tel que

$$
\forall j \geq 1 \quad j^{2(s-1)} \leq a^{2} j^{2 s}+b^{2}
$$


Donc

$$
\begin{aligned}
& \left\|T_{0}^{s-1} f\right\|_{L^{2}\left(\mathbb{R}^{d}\right)}^{2} \leq C a^{2} \sum_{(j, \ell)} j^{2 s}\left\langle f, h_{j, \ell}\right\rangle^{2}+C b^{2} \sum_{(j, \ell)}\left\langle f, h_{j, \ell}\right\rangle^{2} \\
& =C a^{2}\left\|T_{0}^{s} f\right\|_{L^{2}\left(\mathbb{R}^{d}\right)}^{2}+C b^{2}\|f\|_{L^{2}\left(\mathbb{R}^{d}\right)}^{2} \\
& \left\|\left(T_{0}+R\right)^{s} f-T_{0}^{s} f\right\|_{L^{2}\left(\mathbb{R}^{d}\right)} \\
& \leq C\left\|T_{0}^{s-1} f\right\|_{L^{2}\left(\mathbb{R}^{d}\right)} \leq C a\left\|T_{0}^{s} f\right\|_{L^{2}\left(\mathbb{R}^{d}\right)}+C b\|f\|_{L^{2}\left(\mathbb{R}^{d}\right)}
\end{aligned}
$$

En choisissant $a<1 / C$, nous pouvons appliquer le théorème de Kato-Rellich, les opérateurs $\left(T_{0}+R\right)^{s}$ et $T_{0}^{s}$ ont le même domaine. Lorsque $s>0$ n'est plus entier, on conclut par interpolation complexe

$$
\begin{aligned}
& \operatorname{Dom}\left(T^{s}\right)=\left[\operatorname{Dom}\left(T^{E(s)}\right), \operatorname{Dom}\left(T^{E(s)+1}\right)\right]_{\theta}= \\
& {\left[\operatorname{Dom}\left(T_{0}^{E(s)}\right), \operatorname{Dom}\left(T_{0}^{E(s)+1}\right)\right]_{\theta}=\operatorname{Dom}\left(T_{0}^{s}\right)}
\end{aligned}
$$

où $\theta=s-E(s) \in[0,1]$.

Si $R \in \mathcal{B C}^{\infty}\left(\mathbb{R}^{d}\right)$, alors $\lim _{\infty}\|x\|^{2}+R(x)=+\infty$ et il est classique que $T:=$ $-\Delta+\|x\|^{2}+R(x)$ est essentiellement auto-adjoint, à résolvante compacte, que son spectre est discret et qu'il est diagonalisable dans une base orthonormée ([RS75, théorème XIII.67] et [BS91, chapitre 3, théorème 1.1]). Quitte à ajouter une constante à $R$, nous pouvons supposer que l'opérateur $T$ est défini positif (en fait l'hypothèse $R \geq-d$ suffit car $d$ est la plus petite valeur propre de $-\Delta+\|x\|^{2}$ ). Par suite, on peut définir spectralement les opérateurs $T^{s}$. La proposition précédente nous donne les informations supplémentaires suivantes

Corollaire 3.3. Si $R \in \mathcal{B C}^{\infty}\left(\mathbb{R}^{d}\right)$ alors les modes propres de $T_{0}+R$ sont dans l'espace de Schwartz $\mathcal{S}\left(\mathbb{R}^{d}\right)$ et pour tout $s>0$ on a $\operatorname{Dom}\left(T_{0}+R\right)^{s}=\widehat{H}^{s}$.

PREUVE. Il suffit de rappeler que $R \widehat{H}^{s}\left(\mathbb{R}^{d}\right) \subset \widehat{H}^{s}\left(\mathbb{R}^{d}\right)$ pour tout réel $s$. C'est clair si $s$ est un entier naturel d'après la proposition 3.1 et la formule de Leibniz. Par interpolation, c'est donc vrai pour $s>0$. Mais par dualité nous avons aussi pour tout $s>0$ :

$$
\begin{aligned}
& \forall(f, g) \in \widehat{H}^{-s}\left(\mathbb{R}^{d}\right) \times \widehat{H}^{s}\left(\mathbb{R}^{d}\right) \quad|\langle R f, g\rangle|=|\langle f, R g\rangle| \\
& \leq\|f\|_{\widehat{H}^{-s}}\|R g\|_{\widehat{H}^{s}} \leq C\|f\|_{\widehat{H}^{-s}}\|g\|_{\widehat{H}^{s}}
\end{aligned}
$$

Cela prouve que $R f$ appartient à $\widehat{H}^{-s}$. Autrement $\operatorname{dit} R \widehat{H}^{s} \subset \widehat{H}^{s}$ pour tout $s \in \mathbb{R}$.

On convient que la notation $a \simeq b$ signifie que le quotient $\frac{a}{b}$ est compris entre deux constantes universelles strictement positives. La proposition suivante est facile.

Proposition 3.4. En reprenant les notations de (6), si $R \in \mathcal{B C}^{\infty}\left(\mathbb{R}^{d}\right)$ et si le spectre de $-\Delta+\|x\|^{2}+R(x)$ est Zoll-localisé, alors nous avons

$$
\forall(j, \ell) \in E^{+} \quad\left\|\phi_{j, \ell}\right\|_{\widehat{H}^{s}} \simeq j^{s / 2}
$$

Nous avons l'équivalence

i) $\sum_{(j, \ell) \in E^{+}} z_{j, \ell} \phi_{j, \ell} \in \widehat{H}^{s}$ 
ii) $\sum_{(j, \ell) \in E^{+}} \lambda_{j, \ell}^{s}\left|z_{j, \ell}\right|^{2}<+\infty$

iii) $\sum_{j \geq 1} j^{s} \sum_{\ell=1}^{d_{j}}\left|z_{j, \ell}\right|^{2}<+\infty$

PREuve. Pour la première estimation, nous avons

$$
\left\|\phi_{j, \ell}\right\|_{\widehat{H}^{s}} \simeq\left\|T^{s / 2} \phi_{j, \ell}\right\|_{L^{2}} \simeq \lambda_{j, \ell}^{s / 2} \simeq j^{s / 2}
$$

La fin découle aisément de l'équivalence

$$
f \in \widehat{H}^{s}\left(\mathbb{R}^{d}\right) \Leftrightarrow\left(f, T^{s / 2} f\right) \in L^{2}\left(\mathbb{R}^{d}\right) \times L^{2}\left(\mathbb{R}^{d}\right)
$$

3.2. Condition de non-résonance. Définissons à présent la condition de non-résonance. Cette condition technique apparaît dans les travaux [GIP09, Ime12, DS04, DS06b, BDGS07] et dans une version un peu différente dans [BG06].

Définition 3.5. Nous dirons qu'une suite $\left(\lambda_{j, \ell}\right)_{E^{+}}$est non-résonante si

$\forall r \geq 3 \quad \forall k \in[[1, r-1]] \quad \exists \delta, \gamma>0 \quad \forall j_{1}, \cdots, j_{r} \in \mathbb{N}^{\star} \quad \forall \ell_{1}, \cdots, \ell_{r} \in \prod_{i=1}^{r}\left[\left[1, d_{j_{i}}\right]\right]$

$$
\left\{j_{1}, \cdots, j_{k}\right\} \neq\left\{j_{k+1}, \cdots, j_{r}\right\} \Rightarrow\left|\sum_{i=1}^{k} \lambda_{j_{i}, \ell_{i}}-\sum_{i=k+1}^{r} \lambda_{j_{i}, \ell_{i}}\right| \geq \frac{\gamma}{\left(j_{3}^{\star}\right)^{\delta}}
$$

où $j_{3}^{\star}$ est le troisième plus grand entier parmi $j_{1}, \cdots, j_{r}$.

Une argumentation due à Delort-Szeftel montre la proposition suivante :

Proposition 3.6. Soit $R \in \mathcal{B C}^{\infty}\left(\mathbb{R}^{d}, \mathbb{R}\right)$ tel que la propriété (Z) est vérifiée. Il existe une application réelle analytique $M:] 0,1[\rightarrow \operatorname{Com}(T)$ telle que $\|M(m)\| \leq$ $C m$ et le spectre de $-\Delta+\|x\|^{2}+R(x)+M(m)$ est non-résonant pour presque tout $m>0$.

Preuve. On note $\left(\lambda_{j, \ell}\right)_{E^{+}}$le spectre de $-\Delta+\|x\|^{2}+R(x)$. Nous allons invoquer un argument de Delort-Szeftel. D'après la proposition 2.2.1 de [DS06a], pour presque tout $m>0$, la suite $\left(\sqrt{\lambda_{j, l}^{2}+m^{2}}\right)_{(j, l)}$ est non-résonante. Remarquons que c'est à ce point que la croissance polynomiale de $d_{j}$ intervient (voir le lemme 2.2.2 de [DS06a]). Puisque la fonction $m \in] 0, \infty\left[\mapsto \lambda_{1,1} \sqrt{m} \in\right] 0, \infty[$ est localement absolument continue, elle conserve les ensembles Lebesgue-négligeables. Ainsi, $\left(\sqrt{\lambda_{j, l}^{2}+m \lambda_{1,1}^{2}}\right)_{(j, l)}$ est non-résonant pour presque tout $m$. Nous définissons alors $M(m)$ l'unique opérateur vérifiant $M(m) \phi_{j, l}=\left(\sqrt{\lambda_{j, l}^{2}+m \lambda_{1,1}^{2}}-\lambda_{j, l}\right) \phi_{j, l}$. Il est immédiat que $M(m)$ commute avec $T$ et est borné de norme $\leq m \lambda_{1,1} / 2$. De plus, $M(m)$ est compact car autoadjoint et vérifie $\lim _{|j|+|l| \rightarrow+\infty}\left\langle M(m) \phi_{j, l}, \phi_{j, l}\right\rangle=0$. Nous vérifions facilement que $M$ est analytique réelle de ]0,1[ à valeurs dans $\mathcal{L}\left(L^{2}\left(\mathbb{R}^{d}\right)\right)$ :

$$
\forall m \in] 0,1\left[\quad M(m) \phi_{j, l}=\lambda_{j, l}\left(\sqrt{1+\frac{m \lambda_{1,1}^{2}}{\lambda_{j, l}^{2}}}-1\right) \phi_{j, l}=\sum_{n \geq 1} m^{n} M_{[n]} \phi_{j, l}\right.
$$


où $M_{[n]}$ est l'unique opérateur borné de $L^{2}\left(\mathbb{R}^{d}\right)$ défini par

$$
\forall(j, l) \in E^{+} \quad M_{[n]} \phi_{j, l}=\left(\begin{array}{c}
n \\
1 / 2
\end{array}\right) \frac{\lambda_{1,1}^{2 n}}{\lambda_{j, l}^{2 n-1}} \phi_{j, l}
$$

Nous avons en outre

$$
\sup _{n \geq 1}\left\|M_{[n]}\right\|=\sup _{n \geq 1}\left|\left(\begin{array}{c}
n \\
1 / 2
\end{array}\right) \lambda_{1,1}\right|=\sup _{n} \frac{(2 n) ! \lambda_{1,1}}{\left(n ! 2^{n}\right)^{2}(2 n-1)}<+\infty
$$

cela prouve l'analyticité de $m \mapsto M(m)=\sum_{n \geq 1} M_{[n]} m^{n}$ sur $] 0,1$. Par construction, le spectre de $T+M(m)$ est non-résonant. La propriété $(\mathrm{Z})$ est immédiate car

$$
\left|\sqrt{\lambda_{j, l}^{2}+m \lambda_{1,1}^{2}}-\lambda_{j, l}\right| \leq \frac{m \lambda_{1,1}^{2}}{\sqrt{\lambda_{j, l}^{2}+m \lambda_{1,1}^{2}}+\lambda_{j, l}} \leq \frac{m \lambda_{1,1}^{2}}{2 \lambda_{j, l}} \leq \frac{C}{j}
$$

Remarque 3.7. Il aurait été bien plus intéressant, au sens physique, de perturber T par un potentiel. Nous estimons qu'une telle construction passe nécessairement par la compréhension d'un problème spectral inverse. Voir la partie 5 pour le cas de la dimension 1.

3.3. Lemme de commutateur et estimées des normes de Lebesgue. Le lemme suivant nous est utile pour démontrer les estimations multilinéaires de

Lemme 3.8. Soient a, $R \in \mathcal{B C}^{\infty}\left(\mathbb{R}^{d}\right)$ et $T=-\Delta+\|x\|^{2}+R(x)$, considérons la suite d'opérateurs continus de l'espace de Schwartz $\mathcal{S}\left(\mathbb{R}^{d}\right)$ définis par la récurrence

$$
A_{0}=a, \quad \forall n \in \mathbb{N} \quad A_{n+1}=\left[A_{n}, T\right]=A_{n} T-T A_{n}
$$

Nous avons une décomposition

$$
A_{n}=\sum_{|\alpha| \leq n} W_{n, \alpha}(a) D^{\alpha}
$$

où $W_{n, \alpha}: \mathcal{S}\left(\mathbb{R}^{d}\right) \rightarrow \mathcal{S}\left(\mathbb{R}^{d}\right)$ est un opérateur linéaire vérifiant

$$
\forall s \in \mathbb{R} \quad\left\|W_{n, \alpha}(a)\right\|_{\widehat{H}^{s}} \leq C_{n, s}|| a \|_{\widehat{H}^{s+2 n-|\alpha|}}
$$

Par conséquent, on a

$$
\forall f \in \mathcal{S}\left(\mathbb{R}^{d}\right) \quad\left\|A_{n}(f)\right\|_{L^{2}} \leq C_{n} \sum_{|\alpha| \leq n}\|a\|_{\widehat{H}^{d+2 n-|\alpha|} \mid}\|f\|_{\widehat{H}^{|\alpha|}}
$$

Preuve. On note $W(x)=\|x\|^{2}+R(x)$. Pour $n=0$ on a $A_{0}=a$ et donc $W_{0,0}=$ Id. On suppose le résultat vrai au rang $n$. Pour tout $f \in \mathcal{S}\left(\mathbb{R}^{d}\right)$, le terme $A_{n+1}(f)=\left[A_{n}, T\right](f)$ s'écrit 


$$
\begin{aligned}
& \Delta\left(A_{n}(f)\right)-A_{n}(\Delta f)+A_{n}(W f)-W A_{n}(f) \\
= & \sum_{|\alpha| \leq n} \Delta\left(W_{n, \alpha}(a)\right) f^{(\alpha)}+2 \sum_{i=1}^{d} \partial_{i} W_{n, \alpha}(a) \partial_{i} f^{(\alpha)} \\
& +\sum_{|\alpha| \leq n} W_{n, \alpha}(a) D^{\alpha}(W f)-W_{n, \alpha}(a) W f^{(\alpha)} \\
= & \sum_{|\alpha| \leq n} \Delta\left(W_{n, \alpha}(a)\right) f^{(\alpha)}+2 \sum_{i=1}^{d} \partial_{i} W_{n, \alpha}(a) \partial_{i} f^{(\alpha)}+ \\
& \sum_{|\alpha| \leq n} \sum_{0<\beta \leq \alpha} \frac{\alpha !}{\beta !(\alpha-\beta) !} W_{n, \alpha}(a) W^{(\beta)} f^{(\alpha-\beta)}
\end{aligned}
$$

Il ne reste plus qu'à vérifier les estimations de type (13) au rang $n+1$.

$$
\begin{aligned}
\left\|\Delta\left(W_{n, \alpha}(a)\right)\right\|_{\widehat{H}^{s}} \leq & \left\|W_{n, \alpha}(a)\right\| \widehat{H}_{\widehat{H}^{s+2}} \leq C_{n, s+2}\|a\|_{\widehat{H}^{s+2+2 n-|\alpha|}} \\
& =C_{n, s+2}\|a\|_{\widehat{H}^{s+2(n+1)-|\alpha|}} \\
\left\|\partial_{i} W_{n, \alpha}(a)\right\|_{\widehat{H}^{s}} \leq & \left\|W_{n, \alpha}(a)\right\|{\widehat{\widehat{H}^{s+1}}} \leq C_{n, s+1}\|a\|_{\widehat{H}^{s+1+2 n-|\alpha|}} \\
& =C_{n, s+1}\|a\|_{\widehat{H}^{s+2(n+1)-(|\alpha|+1)}} \\
\left\|W^{(\beta)} W_{n, \alpha}(a)\right\|_{\widehat{H}^{s}} \leq & C\left\|W_{n, \alpha}(a)\right\|_{\widehat{H}^{s+1}} \leq C_{n, s+1}\|a\|_{\widehat{H}^{s+1+2 n-|\alpha|}} \\
\leq & C_{n, s+1}\|a\|_{\widehat{H}^{s+2(n+1)-|\alpha-\beta|}}
\end{aligned}
$$

À la dernière étape, l'inégalité $\left\|W^{(\beta)} W_{n, \alpha}(a)\right\|_{\widehat{H}^{s}} \leq C\left\|W_{n, \alpha}(a)\right\|_{\widehat{H}^{s+1}}$ vient de $\beta \neq$ 0 . En se rappelant des inclusions continues $\widehat{H}^{d}\left(\mathbb{R}^{d}\right) \subset H^{d}\left(\mathbb{R}^{d}\right) \subset L^{\infty}\left(\mathbb{R}^{d}\right)$, nous obtenons l'estimation (14):

$$
\left\|A_{n}(f)\right\|_{L^{2}} \leq \sum_{|\alpha| \leq n}\left\|W_{n, \alpha}(a)\right\|_{L^{\infty}}\|f\|_{H^{\alpha}} \leq C_{n} \sum_{|\alpha| \leq n}\|a\|_{\widehat{H}^{d+2 n-\alpha}}\|f\|_{\widehat{H}^{\alpha}}
$$

Nous avons aussi besoin des estimations des normes de Lebesgue des modes propres de $-\Delta+\|x\|^{2}+R(x)$ qui se trouvent dans [KT05] (on y examinera les théorème 4 et corollaire 3.2 ).

Théorème 3.9. (Koch-Tataru) Soient $W \in \mathcal{C}^{\infty}\left(\mathbb{R}^{d}, \mathbb{R}\right)$ un potentiel qui vérifie

$$
W(x) \simeq\|x\|^{2}, \quad\|\nabla W(x)\| \simeq\|x\|, \quad\left\|\partial_{x}^{2} W\right\| \simeq 1
$$

et $\phi \in L^{2}\left(\mathbb{R}^{d}\right) \backslash\{0\}$ un mode propre de $-\Delta+W$ associé à la valeur propre $\lambda>0$, alors

$$
\|\phi\|_{L^{p}\left(\mathbb{R}^{d}\right)} \lesssim \lambda^{\rho_{d}(p)}\|\phi\|_{L^{2}\left(\mathbb{R}^{d}\right)}
$$

où $\rho_{d}(p)$ est défini comme suit si $d \geq 2$ :

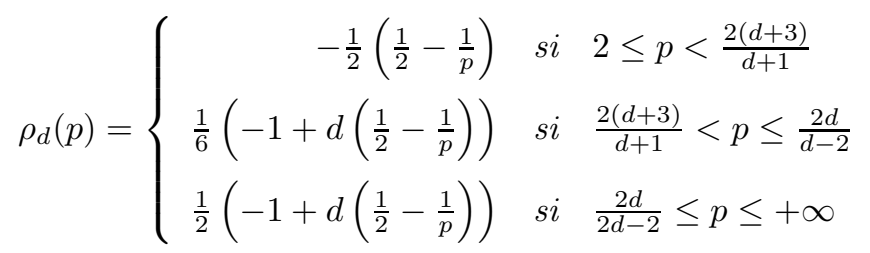


Et comme ceci dans le cas $d=1$ :

$$
\rho_{1}(p)=\left\{\begin{array}{rll}
-\frac{1}{2}\left(\frac{1}{2}-\frac{1}{p}\right) & \text { si } & 2 \leq p<4 \\
\frac{1}{6}\left(-1+\frac{1}{2}-\frac{1}{p}\right) & \text { si } & 4<p \leq+\infty
\end{array}\right.
$$

Remarque 3.10. Le cas $(d, p)=(1,4)$, absent de [KT05], est traité dans le corollaire 5.6 pour les fonctions de Hermite, i.e. si $W(x)=\|x\|^{2}$.

3.4. Estimations multilinéaires des modes propres. On indexe les modes propres de $-\Delta+\|x\|^{2}+R(x)$ en une suite $\left(\phi_{j, \ell}\right)_{(j, \ell) \in E^{+}}$associée à $\left(\lambda_{j, \ell}\right)_{E^{+}}$.

Proposition 3.11. Soit $R \in \mathcal{B C}^{\infty}\left(\mathbb{R}^{d}\right)$ et supposons que le spectre de $T=$ $-\Delta+\|x\|^{2}+R(x)$ est Zoll-localisé, nous affirmons que les intégrales-produits des modes-propres de $T$ vérifient les estimations suivantes : pour tout entier $k \geq 3$, il existe des constantes $\nu \in \mathbb{R}, \beta>0$ telles que

$$
\begin{gathered}
\forall N \geq 1 \quad \forall j_{1} \geq \cdots \geq j_{k} \geq 1 \quad \forall\left(\ell_{1}, \cdots, \ell_{k}\right) \in \prod_{i=1}^{k}\left[\left[1, d_{j_{i}}\right]\right] \\
\left|\int_{\mathbb{R}^{d}} \phi_{j_{1}, \ell_{1}}(x) \cdots \phi_{j_{k}, \ell_{k}}(x) d x\right| \leq C(k, N) \frac{j_{3}^{\nu}}{j_{1}^{\beta}}\left(\frac{\sqrt{j_{2} j_{3}}}{\sqrt{j_{2} j_{3}}+j_{1}-j_{2}}\right)^{N}
\end{gathered}
$$

Dans la suite nous dirons que $T$ vérifie la propriété $(I-P)$. Si $d=1$ et $R \in L^{1}(\mathbb{R})$ alors la propriété (I-P) est automatique.

La démonstration se fait en trois étapes.

\section{Première étape}

Nous affirmons que pour tout $k \geq 3$, il existe des constantes $\beta, \nu, C>0$ qui dépendent de $k$ telles que

$$
\begin{gathered}
\forall j_{1} \geq \cdots \geq j_{k} \geq 1 \quad \forall\left(l_{1}, \cdots, l_{k}\right) \in \prod_{i=1}^{k}\left[\left[1, d_{i}\right]\right] \\
\left|\int_{\mathbb{R}^{d}} \phi_{j_{1}, \ell_{1}}(x) \cdots \phi_{j_{k}, \ell_{k}}(x) d x\right| \leq C(k) \frac{j_{3}^{\nu}}{j_{1}^{\beta}}
\end{gathered}
$$

Si $d=1$, il suffit d'appliquer l'inégalité de Cauchy-Schwarz et d'utiliser les estimations des normes de Lebesgue pour $(d, p)=(1,+\infty)$.

$$
\begin{aligned}
& \left|\int_{\mathbb{R}^{d}} \phi_{j_{1}, \ell_{1}}(x) \cdots \phi_{j_{k}, \ell_{k}}(x) d x\right| \leq \prod_{i=1}^{k-2}\left\|\phi_{j_{i}, \ell_{i}}\right\|_{L^{\infty}(\mathbb{R})} \\
& \leq \frac{C^{k-2}}{\lambda_{j_{1}, \ell_{1}}{ }^{1 / 12} \cdots \lambda_{j_{k-2}, \ell_{k-2}}{ }^{1 / 12}} \leq \frac{C(k)}{j_{1}^{1 / 12}}
\end{aligned}
$$

La dernière majoration découle de l'appartenance $\lambda_{j_{1}, \ell_{1}} \in K_{j_{1}}$ (voir (7)). Si $d \geq 2$, il s'agit d'appliquer l'inégalité de Hölder correctement. Choisissons $p_{1}=p_{2} \in$ ] $2, \frac{2(d+3)}{d+1}\left[\right.$ et $p_{3}, \cdots, p_{k}>2$ arbitraires de sorte que $\sum \frac{1}{p_{i}}=1$. Comme $\rho\left(p_{1}\right)=$ $\rho\left(p_{2}\right)<0$, nous avons :

$$
\left|\int_{\mathbb{R}^{d}} \phi_{j_{1}, \ell_{1}}(x) \cdots \phi_{j_{k}, \ell_{k}}(x) d x\right| \leq \prod_{i=1}^{k}\left\|\phi_{j_{i}, \ell_{i}}\right\|_{L^{p_{i}\left(\mathbb{R}^{d}\right)}} \leq \frac{C(k)}{\left(\lambda_{j_{1}, \ell_{1}} \lambda_{j_{2}, \ell_{2}}\right)^{-\rho\left(p_{1}\right)}} \times \prod_{i=3}^{k} \lambda_{j_{i}, \ell_{i}}^{\rho\left(p_{i}\right)}
$$


La conclusion découle aisément du théorème 3.9 et de l'équivalent $\lambda_{j, \ell} \simeq 2 j$.

\section{Deuxième étape}

Nous faisons maintenant une disjonction de cas.

Premier cas. Si $j_{1}-j_{2} \leq \sqrt{j_{2} j_{3}}$ alors on a

$$
\frac{\sqrt{j_{2} j_{3}}}{j_{1}-j_{2}+\sqrt{j_{2} j_{3}}} \geq \frac{1}{2}
$$

L'estimation (16) implique trivialement (15).

Second cas. Si $j_{1}-j_{2}>\sqrt{j_{2} j_{3}} \geq 1$ alors nous allons appliquer le lemme 3.8 à $a=\phi_{j_{3}, \ell_{3}} \cdots \phi_{j_{k}, \ell_{k}}$. On notera que $a \in \mathcal{S}\left(\mathbb{R}^{d}\right)$ en vertu du corollaire 3.3. L'intérêt des commutateurs apparaît ici :

$$
\begin{aligned}
\int_{\mathbb{R}^{d}} \phi_{j_{1}, \ell_{1}}(x) \cdots \phi_{j_{k}, \ell_{k}}(x) d x & =\int_{\mathbb{R}^{d}} \phi_{j_{1}, \ell_{1}}(x) \phi_{j_{2}, \ell_{2}}(x) a(x) d x \\
& =\frac{1}{\lambda_{j_{1}}-\lambda_{j_{2}}} \int_{\mathbb{R}^{d}} a \phi_{j_{2}, \ell_{2}} T \phi_{j_{1}, \ell_{1}}-a \phi_{j_{1}, \ell_{1}} T \phi_{j_{2}, \ell_{2}} d x \\
& =\frac{1}{\lambda_{j_{1}}-\lambda_{j_{2}}} \int_{\mathbb{R}^{d}} \phi_{j_{1}, \ell_{1}} T\left(a \phi_{j_{2}, \ell_{2}}\right)-\phi_{j_{1}, \ell_{1}} a T \phi_{j_{2}, \ell_{2}} d x \\
& =\frac{1}{\lambda_{j_{1}}-\lambda_{j_{2}}} \int_{\mathbb{R}^{d}} \phi_{j_{1}, \ell_{1}}[T, a] \phi_{j_{2}, \ell_{2}} d x
\end{aligned}
$$

Une récurrence immédiate sur $n$ et l'estimation (14) amènent à

$$
\begin{aligned}
& \left|\int_{\mathbb{R}^{d}} \phi_{j_{1}, \ell_{1}}(x) \cdots \phi_{j_{k}, \ell_{k}}(x) d x\right|=\left|\lambda_{j_{1}}-\lambda_{j_{2}}\right|^{-2 n}\left|\int_{\mathbb{R}^{d}} \phi_{j_{1}, \ell_{1}} A_{2 n}\left(\phi_{j_{2}, \ell_{2}}\right) d x\right| \\
& \leq C\left|j_{1}-j_{2}\right|^{-2 n}\left\|A_{2 n} \phi_{j_{2}, \ell_{2}}\right\|_{L^{2}\left(\mathbb{R}^{d}\right)} \\
& \leq C_{n}\left|j_{1}-j_{2}\right|^{-2 n} \sum_{|\alpha| \leq 2 n}\|a\|_{\widehat{H}^{d+4 n-|\alpha|}}|| \phi_{j_{2}, \ell_{2}} \|_{\widehat{H}^{|\alpha|}} \\
& \leq C_{n}\left|j_{1}-j_{2}\right|^{-2 n} \sum_{|\alpha| \leq 2 n}^{|\alpha| \leq 2 n}|| a \|_{\widehat{H}^{d+4 n-|\alpha|}} j_{2}^{|\alpha| / 2}
\end{aligned}
$$

La dernière majoration vient de l'asymptotique (11). Pour dominer $\|a\|_{\widehat{H}^{s}}$, avec $s>$ 0, nous invoquons l'inégalité suivante (voir [AG91, page 98, chapitre 2, Proposition 2.1.1])

$$
\forall u, w \in H^{s}\left(\mathbb{R}^{d}\right) \cap L^{\infty}\left(\mathbb{R}^{d}\right) \quad\|u w\|_{H^{s}} \leq C(s)\left(\|u\|_{H^{s}}\|w\|_{L^{\infty}}+\|u\|_{L^{\infty}}\|w\|_{H^{s}}\right)
$$

Mais comme $\|u\|_{\widehat{H}^{s}} \simeq\|u\|_{H^{s}}+\left\|u(x)\langle x\rangle^{s}\right\|_{L^{2}}$, nous obtenons facilement

$$
\forall u, w \in \widehat{H}^{s}\left(\mathbb{R}^{d}\right) \cap L^{\infty}\left(\mathbb{R}^{d}\right) \quad\|u w\|_{\widehat{H}^{s}} \leq C(s)\left(\|u\|_{\widehat{H}^{s}}\|w\|_{L^{\infty}}+\|u\|_{L^{\infty}}\|w\|_{\widehat{H}^{s}}\right)
$$

Associés à l'inégalité $\left\|\phi_{j, \ell}\right\|_{\widehat{H}^{s}} \simeq j^{s / 2}$, le théorème 3.9 et une récurrence fournissent l'inégalité

$$
\|a\|_{\widehat{H}^{s}}=\left\|\phi_{j_{3}, \ell_{3}} \cdots \phi_{j_{k}, \ell_{k}}\right\|_{\widehat{H}^{s}} \leq C(k) j_{3}^{s / 2+\nu}
$$


où $\nu=(k-3) \max \left(\rho_{d}(\infty), 0\right)$. Par suite, il vient

$$
\begin{aligned}
\left|\int_{\mathbb{R}^{d}} \phi_{j_{1}, \ell_{1}}(x) \cdots \phi_{j_{k}, \ell_{k}}(x) d x\right| & \leq C_{n, k}\left|j_{1}-j_{2}\right|^{-2 n} \sum_{|\alpha| \leq 2 n} j_{3}^{\nu+d / 2+2 n-|\alpha| / 2} j_{2}^{|\alpha| / 2} \\
& \leq C_{n, k} \frac{j_{3}^{\nu+d / 2+2 n}}{\left|j_{1}-j_{2}\right|^{2 n}} \sum_{|\alpha| \leq 2 n}\left(\frac{j_{2}}{j_{3}}\right)^{|\alpha| / 2} \\
& \leq C_{n, k} j_{3}^{\nu+d / 2} \frac{\left(j_{2} j_{3}\right)^{n}}{\left|j_{1}-j_{2}\right|^{2 n}} \\
& \leq C_{n, k} j_{3}^{\nu+d / 2}\left(\frac{\sqrt{j_{2} j_{3}}}{\sqrt{j_{2} j_{3}}+\left|j_{1}-j_{2}\right|}\right)^{2 n}
\end{aligned}
$$

La dernière majoration utilise la condition $j_{1}-j_{2}>\sqrt{j_{2} j_{3}}$. En notant $B$ le dernier majorant et $A$ le membre droit de (16), nous obtenons la propriété (I-P), c'est-à-dire l'inégalité (15), avec l'inégalité $\min (A, B) \leq \sqrt{A B}$.

Troisième étape

Il ne reste plus qu'à traiter la dernière partie de la proposition, c'est-à-dire le cas $d=1$. Le théorème 1.2 de [KKP05] implique le suivant

Théorème 3.12. (Klein-Korotyaev-Pokrovoski) Soit $R: \mathbb{R} \rightarrow \mathbb{R}$ une fonction lipschitzienne à primitive bornée alors le spectre de $-\partial_{x}^{2}+x^{2}+R(x)$ est Zoll-localisé. De manière précise, si on note $\left(\lambda_{j}\right)_{j \geq 1}$ la suite strictement croissante des valeurs propres alors on a

$$
\forall j \geq 1 \quad\left|\lambda_{j}-2 j+1\right| \leq C j^{-1 / 4}
$$

Si de plus $R$ est impaire, alors on peut remplacer le majorant par $C j^{-1 / 3}$.

En fait le théorème précédent est énoncé dans [KKP05] avec l'hypothèse supplémentaire que la fonction $R$ est bornée, mais cela est conséquence de l'inégalité de Kolmogorov :

$$
\sup _{x \in \mathbb{R}}|R(x)| \leq 2 \sqrt{\sup _{x \in \mathbb{R}}\left|R^{\prime}(x)\right| \times \sup _{x \in \mathbb{R}}\left|\int_{0}^{x} R(t) d t\right|}
$$

Donc, si $R \in \mathcal{B C}^{\infty}(\mathbb{R})$ est intégrable alors $-\partial_{x}^{2}+x^{2}+R(x)$ vérifie $(\mathrm{Z})$, et donc (I-P) grâce à ce qui précède.

\section{Modèle hamiltonien}

4.1. Forme hamiltonienne. Nous pouvons passer à la preuve du théorème 2.2. Nous mettons l'EDP (8) sous forme hamiltonienne. Définissons

$$
E=\left\{(j, \ell) \in \mathbb{Z}^{\star} \times \mathbb{N}^{\star},(|j|, \ell) \in E^{+}\right\}
$$

Considérons l'espace des phases

$$
\mathcal{P}_{s}=\left\{\left(z_{j, \ell}\right) \in \mathbb{C}^{E},\|z\|_{s}:=\left(\sum_{j \in \mathbb{Z}^{\star}}|j|^{s} \sum_{\ell=1}^{d_{|j|}}\left|z_{j, \ell}\right|^{2}\right)^{1 / 2}<+\infty\right\}
$$

que nous munissons de la structure symplectique donnée par la forme

$$
\forall x, y \in E \quad \omega(x, y)=\sum_{j \geq 1} \sum_{\ell=1}^{d_{|j|}} x_{j, \ell} y_{-j, \ell}-x_{-j, \ell} y_{j, \ell}
$$


La somme précédente sera convergente dès lors que l'on choisira $s>0$. Nous noterons aussi le crochet de Poisson de deux fonctions $f, g: \mathcal{P}_{s} \rightarrow \mathbb{R}$ :

$$
\{f, g\}=\sum_{(j, \ell) \in E^{+}} \partial_{j, \ell} f \times \partial_{-j, \ell} g-\partial_{-j, \ell} f \times \partial_{j, \ell} g
$$

Soit $\psi$ une solution de l'équation (8) dans l'espace $\mathcal{C}^{0}\left(I, \widehat{H}^{s}\right)$ où $I$ est un voisinage de $0 \in \mathbb{R}$. Nous allons désolidariser $\psi$ de sa conjuguée en posant $\psi_{1}=\psi$ et $\psi_{2}=\bar{\psi}$. Ainsi, (8) équivaut à la paire d'équations

$$
\left\{\begin{aligned}
i \partial_{t} \psi_{1} & =\left(-\Delta+\|x\|^{2}+R(x)+M(m)\right) \psi_{1}+\partial_{2} g\left(\psi_{1}, \psi_{2}\right) \\
-i \partial_{t} \psi_{2} & =\left(-\Delta+\|x\|^{2}+R(x)+M(m)\right) \psi_{2}+\overline{\partial_{2} g\left(\psi_{1}, \psi_{2}\right)}
\end{aligned}\right.
$$

Nous allons résoudre cette EDP avec la condition initiale $\psi_{1}(0, \cdot)=\overline{\psi_{2}(0, \cdot)}$. Comme les deux équations précédentes sont conjuguées l'une de l'autre, on aura l'égalité suivante sur tout le temps d'existence

$$
\forall t \quad \psi_{1}(t, \cdot)=\overline{\psi_{2}(t, \cdot)}
$$

L'holomorphie de $g$ et la propriété $g(a, \bar{a}) \in \mathbb{R}$, valable pour tout $a \in \mathbb{C}$, prouvent que le système précédent se ramène en fait à

$$
\left\{\begin{aligned}
i \partial_{t} \psi_{1} & =\left(-\Delta+\|x\|^{2}+R(x)+M(m)\right) \psi_{1}+\partial_{2} g\left(\psi_{1}, \psi_{2}\right) \\
-i \partial_{t} \psi_{2} & \left.=\left(-\Delta+\|x\|^{2}+R(x)+M(m)\right) \psi_{2}+\partial_{1} g\left(\psi_{1}, \psi_{2}\right)\right)
\end{aligned}\right.
$$

Nous transférons ces deux équations sur les modes propres. Autrement dit, nous posons

$$
\psi_{1}(t, x)=\sum_{j, \ell \in E^{+}} z_{j, \ell}(t) \phi_{j, \ell}(x), \quad \psi_{2}(t, x)=\sum_{j, \ell \in E^{+}} z_{-j, \ell}(t) \phi_{j, \ell}(x)
$$

Rappelons que les modes propres $\phi_{j, \ell}$ peuvent être choisis à valeurs réelles, si bien que la propriété de conjugaison (18) s'exprime

$$
\forall j, \ell, t \quad z_{-j, \ell}(t)=\overline{z_{j, \ell}}(t)
$$

Nous définissons naturellement

$$
\mathcal{P}_{s}^{+}=\left\{\left(z_{j, \ell}\right) \in \mathcal{P}_{s}, \quad \forall(j, \ell) \in E^{+} \quad z_{j,-\ell}=\overline{z_{j, \ell}}\right\}
$$

et nous cherchons donc une solution à valeurs dans $\mathcal{P}_{s}^{+}$. En notant $\left(-\Delta+\|x\|^{2}+\right.$ $R(x)+M(m)) \phi_{j, \ell}=\omega_{j, \ell} \phi_{j, \ell}$ les équations aux valeurs propres, on est ramené au système suivant

$$
\forall j, \ell \in E^{+}\left\{\begin{aligned}
i z_{j, \ell}^{\prime}(t) & =\omega_{j, \ell} z_{j, \ell}(t)+\int_{\mathbb{R}^{d}} \partial_{2} g\left(\psi_{1}, \psi_{2}\right) \phi_{j, \ell} d x \\
-i z_{-j, \ell}^{\prime}(t) & =\omega_{j, \ell} z_{-j, \ell}(t)+\int_{\mathbb{R}^{d}} \partial_{1} g\left(\psi_{1}, \psi_{2}\right) \phi_{j, \ell} d x
\end{aligned}\right.
$$

En notant $z$ la fonction $t \in I \mapsto\left(z_{j, \ell}(t)\right)_{E}$, la proposition 3.4 nous donne

$$
z \in \mathcal{P}_{s} \quad \Leftrightarrow \quad\left(\psi_{1}, \psi_{2}\right) \in \widehat{H}^{s} \times \widehat{H}^{s}
$$


Il ne reste plus qu'à interpréter les équations (21) comme une seule équation hamiltonienne en $z$. Définissons le hamiltonien libre $H_{0}$ et la perturbation $P$ pour tout $z \in \mathcal{P}_{s}:$

$$
\begin{aligned}
H_{0}(z) & =\sum_{(j, \ell) \in E^{+}} \omega_{j, \ell} z_{j, \ell} z_{-j, \ell} \\
P(z) & =\int_{\mathbb{R}} g\left(\sum_{(j, \ell) \in E^{+}} z_{j, \ell} \phi_{j, \ell}(x), \sum_{(j, \ell) \in E^{+}} z_{-j, \ell} \phi_{j, \ell}(x)\right) d x
\end{aligned}
$$

Vérifions que $H_{0}$ et $P$ sont bien définis sur tout $\mathcal{P}_{s}$ pour $s \gg 1$.

$$
\left|H_{0}(z)\right| \leq C \sum_{(j, \ell) \in E^{+}}|j|\left(\left|z_{j, \ell}\right|^{2}+\left|z_{-j, \ell}\right|^{2}\right)=C \sum_{(j, \ell) \in E}|j|^{s}\left|z_{j, \ell}\right|^{2}
$$

Ainsi $H_{0}$ est un polynôme quadratique de $\mathcal{P}_{s}$. Occupons-nous de $g$. Comme $z \in \mathcal{P}_{s}$, les fonctions $\psi_{1}=\sum_{(j, \ell)} z_{j, \ell} \phi_{j, \ell}$ et $\psi_{2}=\sum_{(j, \ell)} z_{j, \ell} \phi_{j, \ell}$ appartiennent à l'espace $\widehat{H}^{s}$, or ce dernier est une algèbre de Banach, non unitaire, pour $s>d / 2$. Comme $g$ : $\mathbb{C}^{2} \rightarrow \mathbb{C}$ est développable en série entière et s'annule en $(0,0)$, la fonction $g\left(\psi_{1}, \psi_{2}\right)$ est parfaitement définie à valeurs dans $\widehat{H}^{s}$ et trivialement holomorphe par rapport à $z \in \mathcal{P}_{s}$ (voir ci-après le développement en série entière). L'injection holomorphe (car linéaire continue) $\widehat{H}^{s} \rightarrow L^{1}$ achève de prouver que $P$ est holomorphe de $\mathcal{P}_{s}$ à valeurs dans $\mathbb{C}$. Le système devient alors

$$
\forall(j, \ell) \in E^{+} \quad\left\{\begin{aligned}
i z_{j, \ell}^{\prime}(t) & =\partial_{z_{-j, \ell}}\left(H_{0}+P\right) \\
-i z_{-j, \ell}^{\prime}(t) & =\partial_{z_{j, \ell}}\left(H_{0}+P\right)
\end{aligned}\right.
$$

Ce qui se simplifie en la forme hamiltonienne suivante

$$
z^{\prime}=X_{H_{0}+P}(z), \quad z(0) \in \mathcal{P}_{s}^{+}
$$

où le gradient symplectique est défini par

$$
X_{f}=i\left(\left(-\partial_{-j, \ell} f\right)_{(j, \ell) \in E \backslash E^{+}},\left(\partial_{-j, \ell} f\right)_{(j, \ell) \in E^{+}}\right)
$$

On remarquera que le hamiltonien $H_{0}+P$ est bien invariant sur les courbes intégrales.

4.2. Application d'un théorème de formes normales. Posons la définition:

Définition 4.1. Un polynôme $Z$ défini sur $\mathcal{P}_{s}$ est en forme normale s'il est de degré pair $2 k$ et de la forme

$$
\forall z \in \mathcal{P}_{s} \quad Z(z)=\sum_{j \in \mathbb{N} \star k} \sum_{\ell_{1}, \cdots, \ell_{k}=1}^{d_{|j|}} \sum_{\ell_{1}^{\prime}, \cdots, \ell_{k}^{\prime}=1}^{d_{|j|}} a_{j, \ell, \ell^{\prime}} \prod_{i=1}^{k} z_{j_{i}, \ell_{i}} z_{-j_{i}, \ell_{i}^{\prime}}
$$

Nous définissons de surcroît "l'action sur le paquet spectral $K_{j}$ " :

$$
\forall z \in \mathcal{P}_{s} \quad J_{j}(z)=\sum_{\ell=1}^{d_{j}} z_{j, \ell} z_{j,-\ell}
$$

Un calcul montre que les crochets de Poisson suivants sont nuls :

$$
\left\{H_{0}, J_{j}\right\}=\left\{Z, J_{j}\right\}=\left\{J_{j}, J_{j^{\prime}}\right\}=0
$$

où $Z$ est en forme normale. En considérant le développement en série entière

$$
\forall x, y \in \mathbb{C} \quad g(x, y)=\sum_{r \geq 3} \frac{1}{r !} \sum_{k=0}^{r} \partial_{1}^{k} \partial_{2}^{r-k} g(0,0) x^{k} y^{r-k}
$$


nous obtenons celui de $P$ sur $\mathcal{P}_{s}$ (voir [Nac69] pour l'holomorphie en dimension infinie) :

$$
\begin{gathered}
P(z)=\sum_{r \geq 3} \frac{1}{r !} \sum_{k=0}^{r} \partial_{1}^{k} \partial_{2}^{r-k} g(0,0) P_{k, r-k}(z) \\
P_{k, r-k}(z)=\int_{\mathbb{R}^{d}}\left(\sum_{(j, \ell) \in E^{+}} z_{j, \ell} \phi_{j, \ell}(x)\right)^{r}\left(\sum_{(j, \ell) \in E^{+}} z_{-j, \ell} \phi_{j, \ell}(x)\right)^{k-r} \\
=\sum_{(j, \ell) \in\left(E^{+}\right)^{r}} z_{j_{1}, \ell_{1}} \cdots z_{j_{k}, \ell_{k}} z_{-j_{k+1}, \ell_{k+1}} \cdots z_{-j_{r}, \ell_{r}} \int_{\mathbb{R}^{d}} \phi_{j_{1}, \ell_{1}}(x) \cdots \phi_{j_{r}, \ell_{r}}(x) d x
\end{gathered}
$$

L'estimation des intégrales-produits, exprimée par la propriété (I-P) (c'est-à-dire l'inégalité (15)), montre que $P$ est dans une classe de fonctions auxquelles on peut appliquer le théorème de formes normales présent dans [GIP09] (théorème 3.9). Signalons que dans ce théorème la condition de non-résonance énoncée semble plus forte, à savoir

$$
\begin{gathered}
\forall r \geq 3 \quad \forall k \in[[1, r-1]] \quad \exists \delta, \gamma>0 \quad \forall j_{1}, \cdots, j_{r} \in \mathbb{N}^{\star} \quad \forall \ell_{1}, \cdots, \ell_{r} \in \prod_{i=1}^{r}\left[\left[1, d_{j_{i}}\right]\right] \\
\left\{j_{1}, \cdots, j_{k}\right\} \neq\left\{j_{k+1}, \cdots, j_{r}\right\} \Rightarrow\left|\sum_{i=1}^{k} \omega_{j_{i}, \ell_{i}}-\sum_{i=k+1}^{r} \omega_{j_{i}, \ell_{i}}\right| \geq \frac{\gamma\left(j_{1}^{\star}-j_{2}^{\star}\right)}{\left(j_{3}^{\star}\right)^{\delta}}
\end{gathered}
$$

où $j_{1}^{\star} \geq j_{2}^{\star} \geq j_{3}^{\star}$ sont les trois plus grands entiers parmi $j_{1}, \cdots, j_{r}$. Mais la preuve de la proposition 3.1 de [GIP09] montre que cela équivaut à notre définition 10 . Le théorème des formes normales énonce ceci : comme les fréquences de $H_{0}=$ $\sum \omega_{j, \ell} z_{j, \ell} z_{-j, \ell}$ constituent une suite non-résonante (proposition 3.6), pour tous entiers $r \geq 3$ et $s \gg 1$, il existe une transformation $\tau$ canonique (i.e. qui conserve les crochets de Poisson), analytique, réelle (i.e. vérifie $\tau\left(\mathcal{P}_{s}^{+}\right)=\mathcal{P}_{s}^{+}$), définie sur un voisinage de l'origine de $\mathcal{P}_{s}$, et qui transforme le hamiltonien $H_{0}+P$ en

$$
\left(H_{0}+P\right) \circ \tau=H_{0}+Z+\mathcal{R}
$$

avec

i) $Z$ est un polynôme en forme normale

ii) $\mathcal{R}$ est négligeable à l'ordre $r:\left\|X_{\mathcal{R}}(z)\right\|_{s} \leq C\|z\|_{s}^{r}$

iii) $\tau$ est proche de l'identité : $\|\tau(z)-z\|_{s} \leq C\|z\|_{s}^{2}$

En fait nous allons appliquer ce théorème à $r+2$ plutôt qu'à $r$. Revenons à l'équation hamiltonienne (22) et effectuons le changement symplectique $z=\tau(\xi)$. La fonction $\xi$ vérifie l'équation hamiltonienne

$$
\xi^{\prime}=X_{H_{0}+Z+R}(\xi), \quad \xi(0) \in \mathcal{P}_{s}^{+}
$$

Examinons alors la fonction

$$
N(\xi(t))=\sum_{(j, \ell) \in E^{+}}|j|^{s} \xi_{j, \ell}(t) \xi_{j,-\ell}(t)=\sum_{j \geq 1}|j|^{s} J_{j}(\xi(t))
$$

Comme $\tau$ est réelle, la condition $\xi(t) \in \mathcal{P}_{s}^{+}$, valable en $t=0$, se transmet pour tout $t$. Si bien que

$$
N(\xi(t))=\sum_{(j, \ell) \in E}|j|^{s}\left|\xi_{j, \ell}(t)\right|^{2}=\|\xi(t)\|_{s}^{2}
$$


Ensuite, par dérivation, il vient

$$
\frac{d}{d t} N(\xi(t))=i\left\{N, H_{0}+Z+\mathcal{R}\right\}
$$

Comme les fonctions $\xi \mapsto \xi_{j, \ell} \xi_{-j, \ell}$ ont des crochets de Poisson deux à deux nuls et comme $N$ et $H_{0}$ ne dépendent que de ces fonctions, nous obtenons

$$
\begin{aligned}
\left|\frac{d}{d t} N(\xi(t))\right| & =|\{N, \mathcal{R}\}(\xi)| \\
& =\left|\sum_{(j, \ell) \in E^{+}} \partial_{j, \ell} N \partial_{-j, \ell} \mathcal{R}-\partial_{-j, \ell} N \partial_{j, \ell} \mathcal{R}\right| \\
& =\left.\left|\sum_{j \geq 1}\right| j\right|^{s}\left(\xi_{-j, \ell} \partial_{-j, \ell} \mathcal{R}-\xi_{j, \ell} \partial_{j, \ell} \mathcal{R}\right) \mid \\
& \leq 2\left(\sum_{j \geq 1} j^{s}\left|\xi_{j, \ell}\right|^{2}\right)^{1 / 2}\left\|X_{\mathcal{R}}(\xi)\right\|_{s} \\
& \leq C\left(\sum_{j \geq 1} j^{s}\left|\xi_{j, \ell}\right|^{2}\right)^{1 / 2}\|\xi(t)\|_{s}^{r+2} \\
& \leq C\|\xi(t)\|_{s}^{r+3}
\end{aligned}
$$

La fonction $\tau$ est définie sur un voisinage de l'origine de $\mathcal{P}_{s}$, disons la boule $B_{\varepsilon}=$ $\left\{\xi,\|\xi\|_{s}<\varepsilon\right\}$. Considérons donc une solution locale de (23) avec condition initiale $\xi(0) \in B_{\varepsilon} \cap \mathcal{P}_{s}^{+}$. Soit $T_{M}$ le plus grand temps sur lequel $\sup _{0 \leq t \leq T_{M}}\|\xi(t)\|_{s} \leq 2 \varepsilon$. Si $T_{M}=\infty$, il n'y a rien à faire. Supposons donc $T_{M}<\infty$. Nous avons

$$
|N(\xi(t))-N(\xi(0))| \leq C t \varepsilon^{r+3}
$$

En faisant tendre $t$ vers $T_{M}$, il vient

$$
4 \varepsilon^{2}-\varepsilon^{2} \leq C T_{M} \varepsilon^{r+3}
$$

Donc $T_{M} \geq C \varepsilon^{-r-1}$. Par ailleurs, puisque $\tau$ est proche de l'identité, nous avons lorsque $\|\xi(0)\|<\varepsilon$ on a $\|z(0)\|=\|\tau(\xi(0))\| \leq C_{\tau} \varepsilon$. La même démarche est valable pour les temps négatifs. Cela achève de prouver le théorème 2.2. Le formalisme hamiltonien permet d'avoir des informations supplémentaires sur les paquets spectraux $K_{j}$. Pour cela, examinons la situation sur l'intervalle temporel d'existence $\left[-C \varepsilon^{-r}, C \varepsilon^{-r}\right]$. La même argumentation nous amène à

$$
\sum_{j \geq 1} j^{s}\left|\frac{d}{d t} J_{j}(\xi(t))\right|=\sum_{j \geq 1} j^{s}\left|\left\{J_{j}, \mathcal{R}\right\}\right| \leq C \varepsilon^{r+3}
$$

et donc

$$
\sum_{j \geq 1} j^{s}\left|J_{j}(\xi(t))-J_{j}(\xi(0))\right| \leq C \varepsilon^{-r} \times \varepsilon^{r+3}=C \varepsilon^{3}
$$

Par ailleurs, $\tau$ est proche de l'identité donc $\|z(t)-\xi(t)\|_{s} \leq C\|z(t)\|_{s}^{2} \leq C \varepsilon^{2}$, ce qui nous amène à

$$
\sum_{j \geq 1} j^{s}\left|J_{j}(z(t))-J_{j}(\xi(t))\right|+\left|J_{j}(z(0))-J_{j}(\xi(0))\right| \leq C \varepsilon^{4}
$$


Et enfin,

$$
\sum_{j \geq 1} j^{s}\left|J_{j}(z(t))-J_{j}(z(0))\right| \leq C \varepsilon^{3}
$$

\section{Potentiels non-résonants en dimension 1 et base duale}

5.1. Suites duales dans un espace de Hilbert. Le couple $(H,\langle\cdot, \cdot\rangle)$ désigne un $\mathbb{R}$-espace de Hilbert séparable muni d'un produit scalaire, typiquement $L^{2}(\mathbb{R})$ ou $\ell^{2}(\mathbb{N})$ muni de leur produit scalaire usuel. Une démarche analogue est valide en considérant des espaces hilbertiens sur le corps $\mathbb{C}$.

Définition 5.1. Deux suites $\left(e_{n}\right)_{n \geq 0}$ et $\left(e_{n}^{\star}\right)_{n \geq 0}$ de $H$ sont duales si $\left\langle e_{n}, e_{m}^{\star}\right\rangle=$ $\delta_{n, m}$ pour tous entiers $n, m \in \mathbb{N}$.

Bien que nous n'en aurons pas besoin, il est évident de vérifier qu'une suite admet une suite duale si et seulement si elle est topologiquement libre.

Proposition 5.2. Soit $\left(e_{n}\right)_{n \geq 0}$ une suite de $H$, les assertions suivantes sont équivalentes :

i) $\left(e_{n}\right)_{n \geq 0}$ admet une suite duale $\left(e_{n}^{\star}\right)_{n \geq 0}$

ii) $\left(e_{n}\right)_{n \geq 0}$ est topologiquement libre, i.e. pour tout entier $n \in \mathbb{N}$ le vecteur $e_{n}$ n'appartient pas à l'adhérence de $\operatorname{Vect}\left(\left\{e_{m}, m \neq n\right\}\right)$

Preuve. i) $\Rightarrow$ ii). Pour tout entier $n$ on a $\left\langle e_{n}^{\star}, e_{n}\right\rangle=1$ et

$$
\operatorname{Vect}\left(\left\{e_{m}, m \neq n\right\}\right) \subset \operatorname{Vect}\left(e_{n}^{\star}\right)^{\perp}
$$

Donc $e_{n}$ n'appartient pas à $\operatorname{Vect}\left(\left\{e_{m}, m \neq n\right\}\right)$.

ii) $\Rightarrow$ i). Il existe $f_{n}$ tel que $\left\langle f_{n}, e_{n}\right\rangle \neq 0$ et $\left\langle f_{n}, e_{m}\right\rangle=0$ pour tout $m \neq n$. On choisit alors $e_{n}^{\star}=\left\langle f_{n}, e_{n}\right\rangle^{-1} f_{n}$.

Voici le critère pratique que nous utiliserons plus loin.

Théorème 5.3. Soit $\left(e_{n}\right)_{n \geq 0}$ une suite libre de $H$, nous notons $\left(\varepsilon_{n}\right)_{n \geq 0}$ la famille orthonormée de Gram-Schmidt de $\left(e_{n}\right)_{n \geq 0}$, c'est-à-dire que l'on a pour tout $n \in \mathbb{N}$

- $\left\langle e_{n}, \varepsilon_{n}\right\rangle>0$

- $\operatorname{Vect}\left(e_{0}, \cdots, e_{n}\right)=\operatorname{Vect}\left(\varepsilon_{0}, \cdots, \varepsilon_{n}\right)$

On note $A=\left(\left\langle e_{m}, \varepsilon_{n}\right\rangle\right)_{(n, m) \in \mathbb{N}^{2}}$ la matrice de passage de $\left(\varepsilon_{n}\right)_{n \geq 0}$ à $\left(e_{n}\right)_{n \geq 0}$, où les indices $n$ et $m$ parcourent respectivement les lignes et les colonnes. Si bien que $A$ est triangulaire supérieure et formellement inversible (i.e. ses éléments diagonaux sont inversibles) et l'on note $A^{-1}=\left(b_{n, m}\right)_{n, m}$. Alors nous avons l'équivalence entre les deux assertions suivantes :

i) la famille $\left(e_{n}\right)_{n \geq 0}$ admet une suite duale

ii) chaque ligne de $A^{-1}$ appartient à $\ell^{2}(\mathbb{N})$ : pour tout $n \in \mathbb{N}$ on a $\sum_{m \in \mathbb{N}} b_{n, m}^{2}<+\infty$

On suppose vraies les deux propriétés précédentes et l'on note $p: H \rightarrow H$ le projecteur orthogonal de $H$ sur $\overline{\operatorname{Vect}\left\{e_{n}, n \in \mathbb{N}\right\}}$. On a l'équivalence

iii) une suite $\left(e_{n}^{\star}\right)_{n}$ est duale de $\left(e_{n}\right)_{n}$

iv) pour tout $n$ on a $p\left(e_{n}^{\star}\right)=\sum_{m \geq 0} b_{n, m} \varepsilon_{m}$ 
Preuve. i) $\Rightarrow$ ii). Soit $\left(f_{n}\right)_{n}$ une suite duale de $\left(e_{n}\right)_{n}$. Pour tout entier $N \geq 0$, on note $p_{N}$ le projecteur orthogonal de $H$ sur $H_{N}:=\operatorname{Vect}\left(e_{0}, \cdots, e_{N}\right)$. Pour tous entiers $(n, m) \in[0, N]^{2}$ on a

$$
\left\langle p_{N}\left(f_{n}\right), e_{m}\right\rangle=\left\langle f_{n}, e_{m}\right\rangle=\delta_{n, m}
$$

Ainsi, $\left(p_{N}\left(f_{n}\right)\right)_{0 \leq n \leq N}$ est une suite duale de $\left(e_{n}\right)_{0 \leq n \leq N}$. Mais comme $\left(e_{n}\right)_{0 \leq n \leq N}$ est une base de l'espace de dimension finie $H_{N}$, la suite $\left(p_{N}\left(f_{n}\right)\right)_{0 \leq n \leq N}$ est unique. Pour déterminer les coordonnées selon $\left(\varepsilon_{n}\right)_{0 \leq n \leq N}$ il s'agit de remarquer que les égalités (24) s'écrivent matriciellement :

$$
\left(\left\langle p_{N}\left(f_{n}\right), \varepsilon_{m}\right\rangle\right)_{0 \leq n, m \leq N} A_{N}=I_{N}
$$

où $A_{N}$ désigne le bloc $[0, N]^{2}$ de $A$. Par inversibilité de $A_{N}$, on a

$$
\left(\left\langle p_{N}\left(f_{n}\right), \varepsilon_{m}\right\rangle\right)_{0 \leq n, m \leq N}=\left(b_{n, m}\right)_{0 \leq n, m \leq N}
$$

Autrement dit, $p_{N}\left(f_{n}\right)=\sum_{0 \leq m \leq N} b_{n, m} \varepsilon_{m}$ et donc

$$
\sum_{m=0} b_{n, m}^{2}=\sup _{N \geq 0}\left\|p_{N}\left(f_{n}\right)\right\|^{2} \leq\left\|f_{n}\right\|^{2}
$$

ii) $\Rightarrow$ i). Pour tout $n$, on note $e_{n}^{\star}=\sum_{m} b_{n, m} e_{m} \in H$. La formule $A^{-1} A=I d$ signifie précisément que $\left\langle e_{n}^{\star}, e_{m}\right\rangle=\delta_{n, m}$.

iii) $\Rightarrow$ iv) Cela a été montré au cours de la preuve i) $\Rightarrow$ ii).

iv) $\Rightarrow$ iii). Il s'agit d'examiner la preuve ii) $\Rightarrow$ i) pour comprendre que l'on a

$$
\left\langle e_{n}^{\star}, e_{m}\right\rangle=\left\langle p\left(e_{n}^{\star}\right), e_{m}\right\rangle=\delta_{n, m}
$$

Remarque 5.4. Le théorème précédent justifie qu'il existe des familles libres $\left(e_{n}\right)_{n \geq 0}$ qui n'ont pas de suite duale. Par exemple, on considère $\left(\varepsilon_{n}\right)_{n \geq 0}$ une base hilbertienne d'un espace de Hilbert, puis $e_{0}=\varepsilon_{0}$ et $e_{n}=\varepsilon_{n}-\varepsilon_{n-1}$ pour tout $n \geq 1$. Pour tout entier $N \geq 0$ le bloc $[0, N]^{2}$ de $A^{-1}$ est

$$
\left(\begin{array}{ccccc}
1 & -1 & 0 & \cdots & 0 \\
0 & \ddots & \ddots & \ddots & \vdots \\
\vdots & \ddots & \ddots & \ddots & 0 \\
\vdots & & \ddots & \ddots & -1 \\
0 & \cdots & \cdots & 0 & 1
\end{array}\right)^{-1}=\left(\begin{array}{ccccc}
1 & \cdots & \cdots & \cdots & 1 \\
0 & \ddots & & & \vdots \\
\vdots & \ddots & \ddots & & \vdots \\
\vdots & & \ddots & \ddots & \vdots \\
0 & \cdots & \cdots & 0 & 1
\end{array}\right)
$$

Ainsi, la matrice $A^{-1}$ ne satisfait pas le point ii).

5.2. Construction explicite d'une base duale. Dans le théorème 2.3, la suite $(\alpha)_{n}$ va intervenir grâce aux propriétés suivantes :

$$
\begin{gathered}
\alpha_{n}=\frac{(2 n) !}{n !^{2} 4^{n}} \simeq \frac{1}{\sqrt{\pi n}} \\
\frac{1}{\sqrt{1-z}}=\sum_{n \geq 0} \alpha_{n} z^{n}, \quad \sqrt{1-z}=1-\sum_{n \geq 1} \frac{\alpha_{n}}{2 n-1} z^{n}
\end{gathered}
$$


Nous nous inspirons de [Wan09] qui suggère de décomposer les produits de fonctions de Hermite $h_{n}(x) h_{m}(x)$ sur les fonctions $h_{2 n}(x \sqrt{2})$. Ce choix est légitime par conservation du terme $e^{-x^{2}}$. Dans le cas $n=m$, nous obtenons une expression étonnamment simple en fonction de $\alpha_{n}$.

Proposition 5.5. Pour tout $n \in \mathbb{N}$ nous avons

$$
h_{n}^{2}(x)=\sum_{k=0}^{n}\left[(2 \pi)^{-1 / 4} \sqrt{\alpha_{k}} \alpha_{n-k}\right] h_{2 k}(x \sqrt{2}) 2^{1 / 4}
$$

Preuve. Pour tout $n \in \mathbb{N}$, la fonction $h_{n}^{2}$ admet une décomposition dans $L^{2}(\mathbb{R})$

$$
h_{n}^{2}(x)=\sum_{k \geq 0} a_{n, k}^{\prime} h_{k}(x \sqrt{2}) 2^{1 / 4}
$$

La parité assure que $a_{n, k}^{\prime}=0$ pour $k$ impair. Ensuite, comme $h_{n}^{2}=c H_{n}^{2}(x) e^{-x^{2}}$ et $h_{k}(x \sqrt{2})=c^{\prime} H_{k}(x \sqrt{2}) e^{-x^{2}}$ on comprend que $a_{n, k}^{\prime}=0$ pour $k>2 n$ par examen des degrés. En changeant nos notations, nous avons obtenu une décomposition

$$
h_{n}^{2}(x)=\sum_{k=0}^{n} a_{n, k} h_{2 k}(x \sqrt{2}) 2^{1 / 4}
$$

avec $a_{n, n} \neq 0$. Cela montre donc la liberté de $\left(h_{n}^{2}\right)_{n \geq 0}$ et que $\left(h_{2 n}(x \sqrt{2}) 2^{1 / 4}\right)_{n \geq 0}$ est la base orthogonale de Gram-Schmidt associée. Par orthogonalité, nous avons

$$
a_{n, k}=\int_{\mathbb{R}} h_{n}^{2}(x) h_{2 k}(x \sqrt{2}) 2^{1 / 4} d x
$$

Le calcul exact de l'intégrale précédente va être obtenue par la méthode des séries génératrices. Rappelons les deux formules suivantes que l'on trouve respectivement dans [Sze75] et [BTT10, lemme A.5] :

$$
\begin{aligned}
\exp \left(2 z x-z^{2}\right) & =\sum_{n \geq 0} \frac{H_{n}(x)}{n !} z^{n} \\
\frac{1}{\sqrt{\pi\left(1-w^{2}\right)}} \exp \left(-\frac{1-w}{1+w} x^{2}\right) & =\sum_{n \geq 0} w^{n} h_{n}^{2}(x)
\end{aligned}
$$

En revenant aux fonctions $h_{n}$ via $(9)$, nous avons pour $(z, w)$ voisin de $(0,0)$ :

$$
\begin{aligned}
\pi^{-1 / 4} \exp \left(2 z x \sqrt{2}-z^{2}-x^{2}\right) & =\sum_{n \geq 0} 2^{n / 2} h_{n}(x \sqrt{2}) \frac{z^{n}}{\sqrt{n !}} \\
\pi^{-1 / 4} \frac{e^{-z^{2}}}{\sqrt{\pi\left(1-w^{2}\right)}} \exp \left(z x \sqrt{8}-\frac{2}{1+w} x^{2}\right) & =\sum_{n, m \geq 0} \frac{2^{m / 2}}{\sqrt{m !}} w^{n} z^{m} h_{n}^{2}(x) h_{m}(x \sqrt{2})
\end{aligned}
$$

Pour faire commuter $\sum \int=\int \sum$, on rappelle que $C=\sup _{m}\left\|h_{m}\right\|_{L^{\infty}}<+\infty$ (voir [KT05]), on a alors

$$
\begin{aligned}
& \sum_{n, m \geq 0} \int_{\mathbb{R}} \frac{2^{m / 2}}{\sqrt{m !}} w^{n} z^{m}\left|h_{n}^{2}(x) h_{m}(x \sqrt{2})\right| d x \\
& \leq C \sum_{n, m \geq 0} w^{n}(z \sqrt{2})^{m} \int_{\mathbb{R}}\left|h_{n}^{2}(x)\right| d x=\frac{C}{(1-w)(1-z \sqrt{2})}<+\infty
\end{aligned}
$$


Par intégration, il vient

$$
\begin{aligned}
& \pi^{-1 / 4} \frac{e^{-z^{2}}}{\sqrt{\pi\left(1-w^{2}\right)}} \frac{\sqrt{\pi(1+w)}}{\sqrt{2}} \exp \left(z^{2}(1+w)\right) \\
& =\sum_{n, m \geq 0} \frac{2^{m / 2}}{\sqrt{m !}} w^{n} z^{m} \int_{\mathbb{R}} h_{n}^{2}(x) h_{m}(x \sqrt{2}) d x \\
\frac{(2 \pi)^{-1 / 4}}{\sqrt{1-w}} \exp \left(z^{2} w\right) & =\sum_{n, m \geq 0} \frac{2^{m / 2}}{\sqrt{m !}} w^{n} z^{m} \int_{\mathbb{R}} h_{n}^{2}(x) h_{m}(x \sqrt{2}) 2^{1 / 4} d x \\
\frac{(2 \pi)^{-1 / 4}}{\sqrt{1-w}} \exp \left(z^{2} w\right) & =\sum_{0 \leq k \leq n} a_{n, k} \frac{2^{k}}{\sqrt{(2 k) !}} w^{n} z^{2 k} \\
(2 \pi)^{-1 / 4} \sum_{\ell, k \geq 0} \frac{\alpha_{\ell}}{k !} w^{\ell+k} z^{2 k} & =\sum_{0 \leq k \leq n} a_{n, k} \frac{2^{k}}{\sqrt{(2 k) !}} w^{n} z^{2 k}
\end{aligned}
$$

La dernière égalité découle de (26). Donc

$$
a_{n, k}=(2 \pi)^{-1 / 4} \frac{\sqrt{(2 k) !}}{k ! 2^{k}} \alpha_{n-k}=(2 \pi)^{-1 / 4} \sqrt{\alpha_{k}} \alpha_{n-k}
$$

Le résultat suivant est sans doute très classique, nous l'indiquons car sa démonstration est rapide et que nous en aurons besoin plus loin dans la proposition 5.8.

Corollaire 5.6. Il existe une constante universelle $C>1$ telle que

$$
\forall n \geq 2 \quad \frac{\sqrt[4]{\ln (n)}}{C n^{1 / 8}} \leq\left\|h_{n}\right\|_{L^{4}} \leq C \frac{\sqrt[4]{\ln (n)}}{n^{1 / 8}}
$$

Preuve. En invoquant la proposition 5.5 et l'égalité de Parseval-Bessel, nous obtenons

$$
\begin{aligned}
\left\|h_{n}\right\|_{L^{4}}^{4} & =\left\|h_{n}^{2}\right\|_{L^{2}}^{2} \\
& =(2 \pi)^{-1 / 2} \sum_{k=0}^{n} \alpha_{k} \alpha_{n-k}^{2} \\
& \simeq \alpha_{n}^{2}+\alpha_{n}+\sum_{0<k<n} \frac{1}{(n-k) \sqrt{k}} \\
& \simeq \frac{1}{n}+\frac{1}{\sqrt{n}}+\sum_{0<k \leq n / 2} \frac{1}{(n-k) \sqrt{k}}+\sum_{n / 2<k<n} \frac{1}{(n-k) \sqrt{k}} \\
& \simeq \frac{1}{n}+\frac{1}{\sqrt{n}}+\frac{1}{n} \sum_{1 \leq k \leq n / 2} \frac{1}{\sqrt{k}}+\frac{1}{\sqrt{n}} \sum_{n / 2<k<n} \frac{1}{n-k} \\
& \simeq \frac{1}{n}+\frac{1}{\sqrt{n}}+\frac{\ln (n)}{n}+\frac{1}{\sqrt{n}} \\
& \simeq \frac{\ln (n)}{\sqrt{n}}
\end{aligned}
$$


Remarque 5.7. Les estimations des normes de Lebesgue $\left\|h_{n}\right\|_{L^{p}(\mathbb{R})}$ se trouvent dans [KT05] pour $p \in] 2, \infty[\backslash\{4\}$ (voir le théorème 3.9 du présent article).

Nous pouvons enfin démontrer le théorème 2.3. Il s'agit d'appliquer le théorème 5.3 à la famille $\left(h_{n}^{2}\right)_{n \geq 0}$. Comme remarqué dans la preuve de la proposition 5.5 , la famille $\left(h_{n}(\cdot \sqrt{2}) 2^{1 / 4}\right)_{n}$ est la famille de Gram-Schmidt de $\left(h_{n}^{2}\right)_{n \geq 0}$. La matrice $A$ de l'énoncé du théorème 5.3 est

$$
(A)_{i, j}=(2 \pi)^{-1 / 4} \sqrt{\alpha_{i}} \alpha_{j-i}
$$

Pour tout entier $n \geq 0$, on note $A_{n}$ le bloc $[0, n]^{2}$ de la matrice infinie $A, \mathcal{N}_{n} \in$ $\mathcal{M}_{n+1}(\mathbb{R})$ définie par $\left(\mathcal{N}_{N}\right)_{i, j}=\delta_{i+1, j}$, nous avons à l'aide des formules $(26)$

$$
\begin{aligned}
& A_{n}=(2 \pi)^{-1 / 4} \operatorname{Diag}\left(\sqrt{\alpha_{0}}, \cdots, \sqrt{\alpha_{n}}\right)\left(\begin{array}{cccc}
\alpha_{0} & \alpha_{1} & \cdots & \alpha_{n} \\
0 & \alpha_{0} & \ddots & \vdots \\
\vdots & \ddots & \ddots & \alpha_{1} \\
0 & \cdots & 0 & \alpha_{0}
\end{array}\right) \\
& A_{n}=(2 \pi)^{-1 / 4} \operatorname{Diag}\left(\sqrt{\alpha_{0}}, \cdots, \sqrt{\alpha_{n}}\right)\left(\alpha_{0} I+\alpha_{1} \mathcal{N}_{n}+\cdots+\alpha_{n} \mathcal{N}_{n}^{n}\right) \\
& A_{n}=(2 \pi)^{-1 / 4} \operatorname{Diag}\left(\sqrt{\alpha_{0}}, \cdots, \sqrt{\alpha_{n}}\right)\left(1-\mathcal{N}_{n}\right)^{-1 / 2} \\
& A_{n}^{-1}=(2 \pi)^{1 / 4}\left(1-\mathcal{N}_{n}\right)^{1 / 2} \operatorname{Diag}\left(\frac{1}{\sqrt{\alpha_{0}}}, \cdots, \frac{1}{\sqrt{\alpha_{n}}}\right) \\
& A_{n}^{-1}=(2 \pi)^{1 / 4}\left(I-\frac{\alpha_{1}}{2 \times 1-1} \mathcal{N}_{n}-\cdots-\frac{\alpha_{n}}{2 n-1} \mathcal{N}_{n}^{n}\right) \operatorname{Diag}\left(\frac{1}{\sqrt{\alpha_{0}}}, \cdots, \frac{1}{\sqrt{\alpha_{n}}}\right) \\
& A_{n}^{-1}=(2 \pi)^{1 / 4}\left(\begin{array}{cccc}
1 & \frac{-\alpha_{1}}{2 \times 1-1} & \cdots & \frac{-\alpha_{n}}{2 \times n-1} \\
0 & 1 & \ddots & \vdots \\
\vdots & \ddots & \ddots & \frac{-\alpha_{1}}{2 \times 1-1} \\
0 & \cdots & 0 & 1
\end{array}\right) \operatorname{Diag}\left(\frac{1}{\sqrt{\alpha_{0}}}, \cdots, \frac{1}{\sqrt{\alpha_{n}}}\right)
\end{aligned}
$$

Ce qui donne pour tous $j \geq i \in[[0, n]]^{2}$ :

$$
\begin{aligned}
& i=j \quad \Rightarrow \quad\left(A_{n}^{-1}\right)_{i, j}=\frac{(2 \pi)^{1 / 4}}{\sqrt{\alpha_{j}}} \\
& j>i \quad \Rightarrow \quad\left(A_{n}^{-1}\right)_{i, j}=\frac{-(2 \pi)^{1 / 4} \alpha_{j-i}}{(2(j-i)-1) \sqrt{\alpha_{j}}}
\end{aligned}
$$

Le point ii) du théorème 5.3 est trivial :

$$
\forall i \in \mathbb{N} \quad \sum_{j>i} \frac{\alpha_{j-i}^{2}}{(2(j-i)-1)^{2} \alpha_{j}} \leq C(i) \sum_{j>i} \frac{1}{j^{5 / 2}}<+\infty
$$


L'estimation des normes $\left\|h_{n}^{\star}\right\|_{L^{\infty}}$ est facile en invoquant l'estimation $\left\|h_{n}\right\|_{L^{\infty}} \leq$ $C n^{-1 / 12}$ (voir [KT05] ou le théorème 3.9) :

$$
\begin{aligned}
\left\|h_{n}^{\star}\right\|_{L^{\infty}} & \leq C n^{1 / 6}+C \sum_{k=n+1}^{\infty} \frac{k^{1 / 6}}{(2(k-n)-1) \sqrt{k-n}} \\
& \leq C n^{1 / 6}+C \sum_{k=n+1}^{\infty} \frac{k^{1 / 6}}{(k-n)^{3 / 2}} \\
& \leq C n^{1 / 6}+C \sum_{k=n+1}^{\infty} \frac{(k-n)^{1 / 6}+n^{1 / 6}}{(k-n)^{3 / 2}} \\
& \leq C n^{1 / 6}
\end{aligned}
$$

Le développement en série orthogonale donné par le point i) du théorème 2.3 prouve directement le point ii) grâce à (25) et à la proposition 3.4.

Par construction, nous avons l'égalité

$$
\forall n \in \mathbb{N} \quad \operatorname{Vect}\left(h_{0}^{2}, \cdots, h_{n}^{2}\right)=\operatorname{Vect}\left(h_{0}(\cdot \sqrt{2}), \cdots, h_{n}(\cdot \sqrt{2})\right)
$$

et a fortiori

$$
\overline{\operatorname{Vect}\left(h_{n}^{2}, n \in \mathbb{N}\right)}=\overline{\operatorname{Vect}\left(h_{2 n}(\cdot \sqrt{2}), n \in \mathbb{N}\right)}
$$

Par ailleurs, comme $\left(h_{n}\right)_{n \geq 0}$ est une base hilbertienne de $L^{2}(\mathbb{R})$ et que $h_{n}(-\bullet)=$ $(-1)^{n} h_{n}$, il est clair que le sous-espace vectoriel fermé engendré par les fonctions $h_{2 n}$ est le sous-espace des fonctions paires $f \in L^{2}(\mathbb{R})$. L'unicité des fonctions $h_{n}^{\star}$ découle de l'équivalence iii) $\Leftrightarrow$ iv) du théorème 5.3 et cela achève la preuve du théorème 2.3. Nous concluons cette partie par la proposition suivante.

Proposition 5.8. Pour toute fonction $R \in L_{\text {pair }}^{2}(\mathbb{R})$, en posant

$$
x_{n}=\int_{\mathbb{R}} R(x) h_{n}^{2}(x) d x
$$

nous avons

$$
\forall n \geq 2 \quad\left|x_{n}\right| \leq C|| R \|_{L^{2}} \frac{\sqrt{\ln (n)}}{n^{1 / 4}}
$$

En interprétant la suite $\left(x_{n}\right)$ comme des coordonnées selon $\left(h_{n}^{\star}\right)_{n \in \mathbb{N}}$ :

$$
R \simeq \sum_{n \geq 0} x_{n} h_{n}^{\star}
$$

nous avons la formule de changement de coordonnées

$\forall z \in]-1,1\left[\quad \sqrt{1-z} \sum_{n \geq 0} x_{n} z^{n}=\frac{1}{(2 \pi)^{1 / 4}} \sum_{n \geq 0}\left(\sqrt{\alpha_{n}} \int_{\mathbb{R}} R(x) h_{2 n}(x \sqrt{2}) 2^{1 / 4} d x\right) z^{n}\right.$

Preuve. La première estimation découle de l'inégalité de Cauchy-Schwarz et de (28) :

$$
\left|x_{n}\right|^{2} \leq \int_{\mathbb{R}}|R(x)|^{2} d x \int_{\mathbb{R}} h_{n}^{4}(x) d x \leq C|| R \|_{L^{2}}^{2} \frac{\ln (n)}{\sqrt{n}}
$$

Pour pouvoir considérer la suite $\left(x_{n}\right)_{n \geq 0}$ comme des coordonnées de $R$ selon la famille $\left(h_{n}^{\star}\right)_{n \geq 0}$, il suffit de justifier par linéarité que la nullité des nombres $x_{n}$ implique la nullité de $R$, ce qui évident par densité de $\mathbb{R}\left[x^{2}\right] e^{-x^{2}}$ dans $L_{\text {pair }}^{2}(\mathbb{R})$. En 
se rappelant que $\sup _{n \geq 0}\left\|h_{n}\right\|_{L^{\infty}}<+\infty$ (voir [KT05]) et en utilisant les formules de la proposition 5.5, nous reconnaissons un produit de Cauchy :

$$
\begin{aligned}
\forall(z, x) \in]-1,1\left[\times \mathbb{R} \sum_{n \geq 0} h_{n}^{2}(x) z^{n}\right. & =\sum_{n \geq 0} \sum_{k=0}^{n}(2 \pi)^{-1 / 4} \sqrt{\alpha_{k}} \alpha_{n-k} h_{2 k}(x \sqrt{2}) 2^{1 / 4} z^{n} \\
& =\frac{1}{(2 \pi)^{1 / 4} \sqrt{1-z}} \sum_{n \geq 0}\left(\sqrt{\alpha_{n}} h_{2 n}(x \sqrt{2}) 2^{1 / 4}\right) z^{n}
\end{aligned}
$$

Et donc pour presque tout $x \in \mathbb{R}$ nous avons l'égalité

$$
\sum_{n \geq 0} R(x) h_{n}^{2}(x) z^{n}=\frac{1}{(2 \pi)^{1 / 4} \sqrt{1-z}} \sum_{n \geq 0}\left(\sqrt{\alpha_{n}} R(x) h_{2 n}(x \sqrt{2}) 2^{1 / 4}\right) z^{n}
$$

Il ne reste plus qu'à intégrer $x$ sur $\mathbb{R}$ et faire commuter $\sum \int=\int \sum$ grâce aux estimations :

$$
\begin{gathered}
\sum_{n \geq 2} \int_{\mathbb{R}}\left|R(x) h_{n}^{2}(x) z^{n}\right| d x \leq C|| R \|_{L^{2}} \sum_{n \geq 2} \frac{\sqrt{\ln (n)}}{n^{1 / 4}}|z|^{n}<+\infty \\
\sum_{n \geq 0}\left(\sqrt{\alpha_{n}}\left|R(x) h_{2 n}(x \sqrt{2})\right| 2^{1 / 4}\right)|z|^{n} d x \leq \|\left. R\right|_{L^{2}} \sum_{n \geq 0} \sqrt{\alpha_{n}}|z|^{n}<+\infty
\end{gathered}
$$

Remarque 5.9. Comme $\left\|h_{n}^{\star}\right\|_{L^{\infty}} \leq C n^{1 / 6}$, si $x_{n}$ décrồt assez rapidement alors le développement formel (29) est une égalité dans $L^{\infty}(\mathbb{R})$.

5.3. Le difféomorphisme de Chelkak-Kargaev-Korotyaev. Nous allons utiliser le théorème spectral inverse 5.15 de Chelkak-Kargaev-Korotyaev, nous renvoyons le lecteur à l'article [CKK04] pour les démonstrations. Mettons en place quelques notations.

Définition 5.10. Soit $R \in \widehat{H}^{1}(\mathbb{R})$, pour tout entier $n \geq 0$ on note $x \mapsto$ $\phi_{n}(R, x) \in L^{2}(\mathbb{R})$ un mode propre réel associé à $\lambda_{n}(\mathbb{R})$. La limite suivante existe et s'appelle la constante normalisante d'indice $n$

$$
\nu_{n}(R):=\lim _{x \rightarrow+\infty} \ln \left|\frac{\phi_{n}(x, R)}{\phi_{n}(-x, R)}\right|
$$

Définition 5.11. Soit $X$ l'espace de Hilbert des suites réelles $x=\left(x_{n}\right)_{n \geq 0}$ telles que la série entière

$$
f_{x}(z):=\sqrt{1-z} \sum_{n \geq 0} x_{n} z^{n}=\sum_{n \geq 0} y_{n} z^{n}
$$

vérifie

$$
\|x\|_{X}:=\sqrt{\sum_{n \geq 0}(1+n)^{3 / 2} y_{n}^{2}}<+\infty
$$

On définit les deux parties suivantes de $X$

$$
\begin{aligned}
& X_{0}=\left\{x \in X, f_{x}(1)=0\right\} \\
& X_{s}=\left\{x \in X, \quad\left(2 n+1+x_{n}\right)_{n} \nearrow\right\}=\left\{x \in X, \quad \forall n \in \mathbb{N} \quad x_{n+1}-x_{n}>-2\right\}
\end{aligned}
$$


Remarque 5.12. La partie $X_{s}$ est convexe. En outre, la formule (31) montre facilement que pour tout $(n, x) \in \mathbb{N} \times X$ on $a\left|x_{n}\right| \leq C_{n}|| x \|_{X}$, donc $X_{s}$ est ouvert. De même, l'inégalité suivante prouve la fermeture de $X_{0}$ :

$$
\forall x \in X \quad\left|f_{x}(1)\right| \leq \sum_{n \geq 0}\left|y_{n}\right| \leq\left(\sum_{n \geq 0} \frac{1}{(1+n)^{3 / 2}}\right)^{1 / 2}\|x\|_{X}
$$

La propriété suivante nous sera utile dans notre construction

Lemme 5.13. Soit $x$ une suite telle que $\left|x_{n}\right| \leq C n^{-3 / 2}$ pour tout $n \in \mathbb{N}^{\star}$ alors $x \in X$.

Preuve. Quitte à changer la constante $C>0$, les formules (31), (25) et (26) amènent aux inégalités suivantes pour tout entier $n \geq 2$

Par suite,

$$
\begin{aligned}
\left|y_{n}\right| & =\left|x_{n}-\sum_{k=1}^{n} x_{n-k} \frac{\alpha_{k}}{2 k-1}\right| \\
& \leq C\left(\frac{1}{n^{3 / 2}}+\frac{\left|x_{0}\right|}{n^{3 / 2}}+\sum_{k=1}^{n-1} \frac{1}{(n-k)^{3 / 2} k^{3 / 2}}\right) \\
& \leq C\left(\frac{1}{n^{3 / 2}}+\sum_{1 \leq k \leq n / 2} \frac{1}{(n-k)^{3 / 2} k^{3 / 2}}\right) \\
& \leq C\left(\frac{1}{n^{3 / 2}}+\frac{2^{3 / 2}}{n^{3 / 2}} \sum_{1 \leq k \leq n / 2} \frac{1}{k^{3 / 2}}\right) \\
& \leq \frac{C}{n^{3 / 2}}
\end{aligned}
$$

$$
\|x\|_{X}^{2} \leq C \sum_{n \geq 0} \frac{(1+n)^{3 / 2}}{(1+n)^{3}}<+\infty
$$

Remarque 5.14. Les formules (31) et (30) permettent d'exprimer précisément $\left(x_{n}\right)_{n \geq 0}$ en fonction de $\left(y_{n}\right)_{n \geq 0}$ :

$$
\begin{aligned}
y_{n} & =x_{n}-\sum_{k=1}^{n} x_{n-k} \frac{\alpha_{k}}{2 k-1} \\
x_{n} & =\sum_{k=0}^{n} y_{n-k} \alpha_{k} \\
\sum_{n \geq 0} x_{n} h_{n}^{\star} & =(2 \pi)^{1 / 4} \sum_{n \geq 0} \frac{y_{n}}{\sqrt{\alpha_{n}}} h_{2 n}(\cdot \sqrt{2}) 2^{1 / 4}
\end{aligned}
$$

Néanmoins la correspondance entre $\left(x_{n}\right)$ et $\left(y_{n}\right)$ possède un défaut de régularité en ce sens qu'elle ne conserve pas les suites à décroissance rapide. Rappelons que $\sum x_{n} h_{n}^{\star}$ appartient à l'espace de Schwartz $\mathcal{S}(\mathbb{R})$ si et seulement si $y_{n}$ est à décroissance rapide. Par exemple si l'on a $0<y_{n} \leq \frac{1}{2^{n}}$ pour tout $n \in \mathbb{N}$, alors une démonstration analogue au lemme 5.13 montre que $x_{n} \simeq \frac{C}{n^{1 / 2}}$. Cela traduit le fait que le produit de Cauchy de deux suites hérite de la propriété de décroissance la plus faible. Voir aussi l'article [Che03] pour d'autres correspondances. 
Nous pouvons maintenant formuler le théorème spectral inverse suivant :

Théorème 5.15. (Chelkak-Kargaev-Korotyaev) L'application suivante est un difféomorphisme réel analytique

$$
\begin{aligned}
\Lambda^{C K K}: \widehat{H}^{1}(\mathbb{R}) & \rightarrow X_{s} \times X_{0} \\
R & \mapsto\left(\lambda_{n}(R)-2 n-1\right)_{n \geq 0},\left(\nu_{n}(R)\right)_{n \geq 0}
\end{aligned}
$$

Le théorème précédent éclaire le résultat de McKean-Trubowitz [MT82] qui justifie l'existence de beaucoup de potentiels $R \in \mathcal{S}(\mathbb{R})$ dont les valeurs propres associées coïncident avec l'oscillateur harmonique. En fait, la démonstration paramètre les potentiels $R \in \mathcal{S}(\mathbb{R})$ par les constantes normalisantes. La démonstration du théorème 5.15 montre en fait aussi le résultat suivant :

Corollaire 5.16. L'application suivante est un difféomorphisme réel analytique

$$
\begin{aligned}
\Lambda_{\text {pair }}^{C K K}: \widehat{H}_{\text {pair }}^{1}(\mathbb{R}) & \rightarrow X_{s} \\
R & \mapsto\left(\lambda_{n}(R)-2 n-1\right)_{n \geq 0}
\end{aligned}
$$

où $\widehat{H}_{\text {pair }}^{1}(\mathbb{R})=\left\{f \in \widehat{H}^{1}(\mathbb{R}), \quad f(x)=f(-x)\right\}$.

Preuve. Au vu de l'énoncé du théorème 5.15 , il s'agit de prouver pour tout $R \in$ $\widehat{H}^{1}(\mathbb{R})$ l'équivalence

$$
R \in \widehat{H}_{\text {pair }}^{1}(\mathbb{R}) \quad \Leftrightarrow \quad \forall n \in \mathbb{N} \quad \nu_{n}(R)=0
$$

Si $R$ est pair alors $\phi_{n}(R, x)$ et $\phi_{n}(R,-x)$ sont deux éléments de $L^{2}(\mathbb{R})$ qui satisfont l'équation

$$
-\phi^{\prime \prime}(x)+x^{2} \phi(x)+R(x) \phi(x)=\lambda_{n}(R) \phi(x)
$$

Comme rappelé plus haut, $\phi_{n}(R, x)$ et $\phi_{n}(R,-x)$ sont proportionnels. L'égalité $\left\|\phi_{n}(R, \cdot)\right\|_{L^{2}(\mathbb{R})}=1=\left\|\phi_{n}(R,-\cdot)\right\|_{L^{2}(\mathbb{R})}$ amène à $\phi_{n}(R, \cdot)= \pm \phi_{n}(R,-\cdot)$ et a fortiori $\nu_{n}(R)=0$. Réciproquement si $R$ est un potentiel tel que $\nu_{n}(R)=0$ pour tout $n \geq 0$, alors la démonstration du théorème 5.15 montre qu'il existe $\widetilde{R} \in \widehat{H}_{\text {pair }}^{1}(\mathbb{R})$ tel que $\lambda_{n}(R)=\lambda_{n}(\widetilde{R})$ pour tout $n \in \mathbb{N}$ (voir la partie 3.3 de [CKK04], page 154). Par ailleurs, $\nu_{n}(R)=\nu_{n}(\widetilde{R})=0$ pour tout $n \in \mathbb{N}$. L'énoncé du théorème [CKK04] assure que $R=\widetilde{R} \in \widehat{H}_{\text {pair }}^{1}(\mathbb{R})$.

Nous allons maintenant donner une représentation naturelle de $\widehat{H}_{\text {pair }}^{1}(\mathbb{R})$ à l'aide de la base duale $\left(h_{n}^{\star}\right)_{n \geq 0}$. phisme

Proposition 5.17. L'application suivante est bien définie et est un isomor-

$$
\begin{aligned}
\Lambda_{c}: \quad X & \rightarrow \widehat{H}^{1}(\mathbb{R}) \\
\left(x_{n}\right)_{n \geq 0} & \mapsto \sum_{n \geq 0} x_{n} h_{n}^{\star}
\end{aligned}
$$

Par conséquent, $\overline{\Lambda_{\text {pair }}^{C K K}}:=\Lambda_{\text {pair }}^{C K K} \circ \Lambda_{c}: X \rightarrow X_{s}$ est un difféomorphisme réel analytique.

Preuve. C'est immédiat grâce à la formule de changement de variable. En effet, en reprenant les formules (32), nous avons

$$
\|x\|_{X}^{2}=\sum_{n \geq 0}(1+n)^{3 / 2} y_{n}^{2} \simeq \sum_{n \geq 0}(1+n)\left(\frac{y_{n}}{\sqrt{\alpha_{n}}}\right)^{2} \simeq\left\|\sum_{n \geq 0} x_{n} h_{n}^{\star}\right\|_{\widehat{H}^{1}}^{2}
$$


Remarque 5.18. En vertu de la formule (5) dont une preuve est présente dans [CKK04], la différentielle de $\overline{\Lambda_{\text {pair }}^{C K K}}$ en 0 est l'opérateur identité Id $: X \rightarrow X$. On retrouve ainsi par inversion locale que $\overline{\Lambda_{\text {pair }}^{C K K}}$ est un difféomorphisme au voisinage de 0. La vraie difficulté, résolue par Chelkak-Kargaev-Korotyaev, réside en réalité dans le fait que $\overline{\Lambda_{\text {pair }}^{C K K}}$ est bien à valeurs dans l'espace $X$.

Le résultat suivant avait été annoncé sans démonstration dans [GIP09] et s'avère utile dans la construction de potentiels non-résonants, la démonstration se trouve en annexe :

Proposition 5.19. Sur un espace probabilisé $\Omega$, nous considérons $\left(Z_{j}\right)_{j \geq 1}$ une suite de variables aléatoires indépendantes identiquement distribuées de loi uniforme sur $\left[-\frac{1}{2}, \frac{1}{2}\right]$, on fixe une entier $k \geq 1$, la suite de terme général

$$
2 j-1+\frac{Z_{j}(\omega)}{j^{k}}
$$

est non-résonante pour presque tout $\omega$ (voir la définition (10) avec $d_{j}=1$ ).

Nous allons pouvoir appliquer le lemme 5.13. À cet effet, on considère une suite $\left(Z_{n}\right)_{n \geq 0}$ de variables aléatoires indépendantes identiquement distribuées de loi uniforme sur $\left[-\frac{1}{2}, \frac{1}{2}\right]$. Pour presque tout $\omega$, la suite $2 n+1+\frac{Z_{n}(\omega)}{(1+n)^{2}}$ est nonrésonante. Le lemme 5.13 justifie que la suite de terme général $\frac{Z_{n}(\omega)}{(1+n)^{2}}$ appartient à $X$. Mais puisque $\left|\frac{Z_{n}(\omega)}{(1+n)^{2}}\right|<1$, nous avons aussi $\left(\frac{Z_{n}(\omega)}{(1+n)^{2}}\right)_{n} \in X_{s}$. Notons $\left(r_{n}(\omega)\right)_{n} \in X$ l'unique élément dont l'image par le difféomorphisme $\overline{\Lambda_{\text {pair }}^{C K K}}$ est $\left(\frac{Z_{n}(\omega)}{(1+n)^{2}}\right)_{n}$. Nous concluons que pour des paramètres génériques $r_{n}$, le spectre de l'opérateur différentiel $-\partial_{x}^{2}+x^{2}+\sum_{n \geq 0} r_{n} h_{n}^{\star}$ est non-résonant.

5.4. Annexe : existence probabiliste de suite non-résonante. La preuve de la proposition 5.19 est très technique et peu transparente, de manière équivalente nous montrons la proposition suivante :

Proposition 5.20. On munit l'espace $\prod_{j}\left[\frac{-1}{2}, \frac{1}{2}\right]$ de la mesure Lebesgue-produit, pour tout entier $k \geq 1$ il existe un ensemble $\mathcal{F}_{k}$ de mesure pleine dont tous les éléments $\left(m_{j}\right)_{j \geq 1}$ sont tels que $\left(2 j-1+\frac{m_{j}}{j^{k}}\right)_{j \geq 1}$ est une suite non-résonante.

Entamons la démonstration technique de la proposition 5.20 par les lemmes suivants

Lemme 5.21. Soient $M>0, c \in \mathbb{R}, \delta>0$ et $\left(a_{1}, \ldots, a_{r}\right) \in \mathbb{Z}^{r} \backslash\{0\}$, il existe une constante $C(r)>0$ telle que

$$
\operatorname{Vol}_{r}\left(\left\{x \in[-M,+M]^{r},\left|\sum_{i=1}^{r} a_{i} x_{i}+c\right|<\delta\right\}\right) \leq C(r) M^{r-1} \delta
$$

où Vol désigne la mesure de Lebesgue sur $\mathbb{R}^{r}$ 
Preuve. On a l'inclusion

$$
\left\{x \in[-M,+M]^{r},\left|\sum_{i=1}^{r} a_{i} x_{i}+c\right|<\delta\right\} \subset\left\{x \in \mathbb{R}^{r}, \sum_{i=1}^{r} x_{i}^{2} \leq r M^{2},\left|\sum_{i=1}^{r} a_{i} x_{i}+c\right|<\delta\right\}
$$

L'inégalité $\left|\sum_{i=1}^{r} a_{i} x_{i}+c\right|<\delta$ signifie que $x$ à une distance $<\frac{\delta}{\sqrt{\sum a_{i}^{2}}}$ de l'hyperplan affine $\sum a_{i} x_{i}+c=0$. Ce dernier rencontre la boule $\sum x_{i}^{2} \leq r M^{2}$ en une boule hyperplane $B^{\prime}$ de volume $(r-1)$-dimensionnel inférieur à $C(r) M^{r-1}$, donc l'ensemble de départ est inclus dans l'ensemble produit $\left.B^{\prime} \times\right]-\frac{\delta}{\sqrt{\sum a_{i}^{2}}}, \frac{\delta}{\sqrt{\sum a_{i}^{2}}}\left[\subset B^{\prime} \times\right]-\delta, \delta[$.

Ensuite, nous avons le

Lemme 5.22. Pour tous entiers naturels non nuls $N$ et $r$ on a

$$
\operatorname{Card}\left\{\alpha \in \mathbb{Z}^{N}, \sum_{i=1}^{N}\left|\alpha_{i}\right| \leq r\right\} \leq C(r) N^{r}
$$

Preuve. Appelons $\xi(N, r)$ le membre gauche. Si $N \leq r$, on a trivialement $\xi(N, r) \leq$ $\xi(r, r)$. On suppose $N \geq r$. Le point clé est que parmi $N$ entiers $\alpha_{1}, \ldots, \alpha_{N}$ vérifiant $\sum\left|\alpha_{i}\right| \leq r$, il y a au plus $r$ entiers non nuls. Autrement dit,

$$
\left\{\alpha \in \mathbb{Z}^{N}, \sum_{i=1}^{N}\left|\alpha_{i}\right| \leq r\right\} \subset \bigcup_{J}\left\{\alpha \in \mathbb{Z}^{n}, \sum_{i=1}^{N}\left|\alpha_{i}\right| \leq r, \forall i \in J \quad \alpha_{i}=0\right\}
$$

où $J$ parcourt les $C_{N-r}^{N}=C_{r}^{N}$ parties de $[[1, N]]$ de cardinal $N-r$. Cela nous amène à $\xi(N, r) \leq \xi(r, r) N^{r} / r$ !.

Dans la suite pour tous $m \in \prod_{j}\left[\frac{-1}{2}, \frac{1}{2}\right]$ et $j \geq 1$ on note $\omega_{j}=2 j-1+\frac{m_{j}}{j^{k}}$.

Proposition 5.23. Soient $\left.r, k \in \mathbb{N}^{\star}, \gamma \in\right] 0,1[$, il existe certaines constantes $C(r, k)>0, \beta(r, \gamma, k) \geq k$ vérifiant ce qui suit. Si $\gamma<1 / C(r, k)$ alors il existe une partie mesurable $F_{r, \gamma}^{\prime} \subset \prod_{j}\left[\frac{-1}{2}, \frac{1}{2}\right]$ de mesure $\geq 1-\gamma C(r, k)$ telle que

$$
\begin{aligned}
& \forall m \in F_{r, \gamma}^{\prime} \quad \forall b \in \mathbb{Z} \quad \forall N \in \mathbb{N}^{\star} \quad \forall \alpha \in \mathbb{Z}^{N} \backslash\{0\} \\
& \sum_{j=1}^{N}\left|\alpha_{j}\right| \leq r \quad \Rightarrow \quad\left|\sum_{j=1}^{N} \alpha_{j} \omega_{j}-b\right| \geq \frac{\gamma}{N^{\beta}}
\end{aligned}
$$

Preuve. Pour tous $\alpha, b$ et $N$ comme dans l'énoncé, introduisons l'ensemble suivant

$$
A_{\alpha, b}=\left\{m \in\left[\frac{-1}{2}, \frac{1}{2}\right]^{N}\left|\sum_{j=1}^{N} \alpha_{j} \omega_{j}-b\right|<\frac{\gamma}{N^{\beta}}\right\}
$$

L'ensemble $\left\{j \in\left[[1, N], \alpha_{j} \neq 0\right\}\right.$ est de cardinal $\leq r$, on considère un ensemble $I(\alpha)$ de cardinal $r$ tel que

$$
\left\{j \in\left[[1, N], \alpha_{j} \neq 0\right\} \subset I_{\alpha} \subset[[1, N]]\right.
$$


Ainsi $A_{\alpha, b}$ se factorise $A_{\alpha, b}=\left[\frac{-1}{2}, \frac{1}{2}\right]^{N-r} \times A_{\alpha, b}^{\prime}$, avec

$$
A_{\alpha, b}^{\prime}=\left\{m \in\left[\frac{-1}{2}, \frac{1}{2}\right]^{I_{\alpha}}\left|\sum_{j \in I_{\alpha}} \alpha_{j} \omega_{j}-b\right|<\frac{\gamma}{N^{\beta}}\right\}
$$

Puisque $\omega_{j}=2 j-1+\frac{m_{j}}{j^{k}}$, en notant la bijection linéaire $\phi: m \in \mathbb{R}^{I_{\alpha}} \mapsto\left(\frac{m_{j}}{j^{k}}\right)_{j} \in$ $\mathbb{R}^{I_{\alpha}}$ on a

$$
\phi\left(A_{\alpha, b}^{\prime}\right) \subset\left\{x \in\left[\frac{-1}{2}, \frac{1}{2}\right]^{I_{\alpha}}\left|\sum_{j=1}^{N} \alpha_{j}\left(2 j-1+x_{j}\right)-b\right|<\frac{\gamma}{N^{\beta}}\right\}
$$

Bien entendu, on identifie $A_{\alpha, b}$ à l'ensemble iso-mesure des $m \in \prod_{j}\left[\frac{-1}{2}, \frac{1}{2}\right]$ tels que $\left(m_{1}, \ldots, m_{N}\right) \in A_{\alpha, b}$. À l'aide du lemme 5.21, on obtient

$$
C(r) \frac{\gamma}{N^{\beta}} \geq \operatorname{det}(\phi) \operatorname{Vol}_{r}\left(A_{\alpha, b}^{\prime}\right) \geq \frac{\operatorname{Vol}_{N}\left(A_{\alpha, b}\right)}{N^{k r}} \geq \frac{\mathbb{P}\left(A_{\alpha, b}\right)}{N^{k r}}
$$

L'idée est de considérer $F_{r, \gamma}^{\prime}$ comme le complémentaire de l'union dénombrable des $A_{\alpha, b}$ quand $N, \alpha$ et $b \in \mathbb{Z}$ sont des paramètres. Déjà le lemme 5.22 nous dit qu'il y a au plus $C(r) N^{r}$ paramètres $\alpha$ à prendre en compte. Quant aux seules valeurs pertinentes de $b$, ce sont celles telles que $A_{\alpha, b} \neq \emptyset$ pour au moins un $\alpha$, et donc

$$
|b| \leq \frac{\gamma}{N^{\beta}}+\left|\sum_{j=1}^{N} \alpha_{j} \omega_{j}\right| \leq 1+2 N r
$$

Pour le choix $\beta=k+2+(k+1) r+1 \geq k$ on a

$$
\mathbb{P}\left(\bigcup_{N, \alpha, b} A_{\alpha, b}\right) \leq \sum_{N, \alpha, b} \mathbb{P}\left(A_{\alpha, b}\right) \leq \gamma C(r) \sum_{N \geq 1} N^{k r-\beta} \times N^{r} \times(1+2 N r) \leq C(r, k) \gamma
$$

Considérons un réel $\Omega>1$ tel que $\frac{j}{\Omega} \leq \omega_{j} \leq \Omega j$ pour tout $j \geq 1$. Nous avons aussi la

Proposition 5.24. Pour tous $\left.\varepsilon=\left(\varepsilon_{1}, \varepsilon_{2}\right) \in\{0,1,-1\}^{2}, r, k \in \mathbb{N}^{\star}, \gamma \in\right] 0,1[$, $i l$ existe $C_{\varepsilon}(r, k)>0, \beta_{\varepsilon}^{\prime}(r, \gamma, k)>0$ et $\left.\left.\gamma_{\varepsilon}^{\prime}(r, \gamma, k) \in\right] 0, \gamma\right]$ et une partie mesurable $F_{\varepsilon, r, \gamma} \subset \prod_{j} \prod_{j}\left[\frac{-1}{2}, \frac{1}{2}\right]$ de mesure $\geq 1-C_{\varepsilon} \gamma$ tels que

$$
\begin{gathered}
\forall m \in F_{\varepsilon, r, \gamma} \quad \forall N \in \mathbb{N}^{\star} \quad \forall \alpha \in \mathbb{Z}^{N} \quad \forall l_{1}>l_{2}>N \quad(\alpha, \varepsilon) \neq(0, \ldots, 0) \\
\sum_{j=1}^{N}\left|\alpha_{j}\right| \leq r \quad \Rightarrow \quad\left|\sum_{j=1}^{N} \alpha_{j} \omega_{j}+\varepsilon_{1} \omega_{l_{1}}+\varepsilon_{2} \omega_{l_{2}}\right| \geq \frac{\gamma_{\varepsilon}^{\prime}}{N^{\beta_{\varepsilon}^{\prime}}}
\end{gathered}
$$

PREUVE.

$\checkmark$ Le cas $\varepsilon=(0,0)$ est une reformulation de la proposition 5.23.

$\checkmark$ Traitons les cas $\varepsilon=\{ \pm 1,0\}$ ou $\varepsilon=\{0, \pm 1\}$. On pose

$$
\begin{gathered}
\left.\left.C_{\varepsilon}(r)=C(r+1), \quad \beta_{\varepsilon}^{\prime}=\beta(r+1, \gamma, k), \quad \gamma_{\varepsilon}^{\prime}=\frac{\gamma}{\left(4 C^{2} r\right)^{\beta_{\varepsilon}^{\prime}}} \in\right] 0, \gamma\right] \\
F_{\varepsilon, r, \gamma}=F_{r+1, \gamma}^{\prime}
\end{gathered}
$$


Pour tout $m \in F_{\varepsilon, r, \gamma}$ et pour tous $N \in \mathbb{N}^{\star}, \alpha \in \mathbb{Z}^{N}$, et $l_{1}>\left(4 \Omega^{2} r\right) N$ alors on a

$$
\left|\sum_{j=1}^{N} \alpha_{j} \omega_{j} \pm \omega_{l_{1}}\right| \geq\left|\omega_{l_{1}}\right|-\left|\sum_{j=1}^{N} \alpha_{j} \omega_{j}\right|
$$

En se rappelant que $\Omega, r$ et $N \geq 1$, on peut minorer

$$
\left|\sum_{j=1}^{N} \alpha_{j} \omega_{j} \pm \omega_{l_{1}}\right| \geq \frac{1}{\Omega} l_{1}-\Omega N r \geq 4 \Omega r N-\Omega r N \geq 1 \geq \frac{\gamma_{\varepsilon}^{\prime}}{N_{\varepsilon}^{\beta_{\varepsilon}^{\prime}}}
$$

Par contre si $N \leq l_{1}<\left(4 \Omega^{2} r\right) N$, alors l'appartenance de $m$ à $F_{r+1, \gamma}^{\prime}$ amène à

$$
\left|\sum_{j=1}^{N} \alpha_{j} \omega_{j} \pm \omega_{l_{1}}\right| \geq \frac{\gamma}{\left(4 C^{2} r\right)^{\beta^{\prime}} N^{\beta^{\prime}}}=\frac{\gamma_{\varepsilon}^{\prime}}{N^{\beta_{\varepsilon}^{\prime}}}
$$

$\checkmark$ Le cas $\varepsilon=(1,1)$ ou $\varepsilon=(-1,-1)$ se traite comme le cas précédent en partant de l'inégalité

$$
\left|\sum_{j=1}^{N} \alpha_{j} \omega_{j} \pm\left(\omega_{l_{1}}+\omega_{l_{2}}\right)\right| \geq \frac{1}{\Omega}\left(l_{1}+l_{2}\right)-\Omega N r
$$

$\checkmark$ Il reste à considérer $\varepsilon=(1,-1)$. Pour $r \in \mathbb{N}^{\star}$, on considère une constante $\mu=\mu(r, \Omega) \geq 1$ telle que

$$
\frac{\mu}{\Omega}-(1+r) \Omega \geq 1
$$

La pertinence de ce choix apparaît plus loin. L'idée est d'appliquer la proposition 5.23 en posant

$$
\begin{gathered}
F_{\varepsilon, r, \gamma}:=F_{r, \gamma}^{\prime} \cap F_{r+2, \gamma}^{\prime}, \quad C_{\varepsilon, r}:=C(r)+C(r+2) \\
\beta_{\varepsilon}^{\prime}(r, \gamma, k)=\frac{1}{k} \beta(r, k, \gamma) \beta(r+2, k, \gamma), \\
\left.\gamma_{\varepsilon}^{\prime}(r, \gamma, k)=\min \left\{\frac{\gamma}{2}, \frac{\gamma}{\left(2 \gamma^{-1} \mu^{k}\right)^{\beta(r+2) / k}}\right\} \in\right] 0, \gamma[
\end{gathered}
$$

Vérifions que cela convient. Déjà on a bien $\mathbb{P}\left(F_{\varepsilon, r, \gamma}\right) \geq 1-(C(r)+C(r+2)) \gamma$. Fixons $m \in F_{\varepsilon, r, \gamma}$ ainsi que $(N, \alpha)$ comme dans l'énoncé. On distingue plusieurs cas

- Si $l_{1} \geq l_{2} \geq\left(2 N^{\beta} \gamma^{-1}\right)^{1 / k}$

$$
\left|\omega_{l_{1}}-\omega_{l_{2}}-\lambda_{l_{1}}+\lambda_{l_{2}}\right|=\left|\frac{m_{l_{1}}}{l_{1}^{k}}-\frac{m_{l_{2}}}{l_{2}^{k}}\right| \leq \frac{\gamma}{2 N^{\beta}}
$$

D'après la proposition 5.23, l'appartenance de $m$ à $F_{r, \gamma}^{\prime}$ donne

$$
\begin{aligned}
& \left|\sum_{j=1}^{N} \alpha_{j} \omega_{j}+\omega_{l_{1}}-\omega_{l_{2}}\right| \geq\left|\sum_{j=1}^{N} \alpha_{j} \omega_{j}+\lambda_{l_{1}}-\lambda_{l_{2}}\right|-\left|\omega_{l_{1}}-\omega_{l_{2}}-\lambda_{l_{1}}+\lambda_{l_{2}}\right| \\
& \geq \frac{\gamma}{N^{\beta}}-\frac{\gamma}{2 N^{\beta}}=\frac{\gamma}{2 N^{\beta}}
\end{aligned}
$$

Et l'on peut minorer par $\gamma_{\varepsilon}^{\prime} N^{-\beta_{\varepsilon}^{\prime}}$ puisque $\beta \geq k$. 
- Si $l_{1} \geq\left(N^{\beta} 2 \gamma^{-1}\right)^{1 / k} \mu \geq\left(N^{\beta} 2 \gamma^{-1}\right)^{1 / k} \geq l_{2}$. Comme $\min \left\{\Omega, \gamma^{-1}, N\right\} \geq 1$ et $\beta \geq k$ (voir la proposition 5.23), on peut minorer

$$
\begin{aligned}
\left|\sum_{j=1}^{N} \alpha_{j} \omega_{j}+\omega_{l_{1}}-\omega_{l_{2}}\right| & \geq \omega_{l_{1}}-\omega_{l_{2}}-\left|\sum_{j=1}^{N} \alpha_{j} \omega_{j}\right| \\
& \geq \frac{l_{1}}{\Omega}-\Omega l_{2}-\Omega r N \\
& \geq \frac{1}{\Omega}\left(N^{\beta} \gamma^{-1} 2\right)^{1 / k} \mu-\Omega\left(N^{\beta} \gamma^{-1} 2\right)^{1 / k}-\Omega r N \\
& \geq\left(\frac{\mu}{\Omega}-\Omega\right)\left(N^{\beta} \gamma^{-1} 2\right)^{1 / k}-\Omega r N \\
& \geq\left(\frac{\mu}{\Omega}-\Omega\right) N-r \Omega N \\
& \geq N\left(\frac{\mu}{\Omega}-\Omega-r \Omega\right) \\
& \geq \frac{\mu}{\Omega}-\Omega-r \Omega \\
& \geq 1
\end{aligned}
$$

- $\left(N^{\beta} 2 \gamma^{-1}\right)^{1 / k} \mu \geq l_{2} \geq l_{1}$ alors l'appartenance $m \in F_{r+2, \gamma}^{\prime}$ s'exprime

$$
\left|\sum_{j=1}^{N} \alpha_{j} \omega_{j}+\omega_{l_{1}}-\omega_{l_{2}}\right| \geq \frac{\gamma}{\left(N^{\beta} 2 \gamma^{-1}\right)^{\beta(r+2, k, \gamma) / k} \mu^{\beta(r+2, \gamma, k)}} \geq \frac{\gamma_{\varepsilon}^{\prime}}{N^{\beta_{\varepsilon}^{\prime}}}
$$

Enfin, on a la proposition suivante.

Proposition 5.25. Pour tous $\left.r, k \in \mathbb{N}^{\star}, \gamma \in\right] 0,1\left[\right.$, il existe $C(r)>0, \beta^{\prime}(r, \gamma)>$ 0 et $\left.\gamma^{\prime}(r, \gamma) \in\right] 0, \gamma\left[\right.$ et une partie mesurable $F_{r, \gamma} \subset \prod_{j}\left[\frac{-1}{2}, \frac{1}{2}\right]$ de mesure $\geq 1-C(r) \gamma$ tels que

$$
\begin{gathered}
\forall m \in F_{r, \gamma} \quad \forall\left\{\varepsilon_{1}, \varepsilon_{2}\right\} \in\{0,1,-1\}^{2} \quad \forall N \in \mathbb{N}^{\star} \\
\forall \alpha \in \mathbb{Z}^{N} \quad \forall l_{1}, l_{2} \in \mathbb{N} \cap[N+1,+\infty[\quad(\alpha, \varepsilon) \neq(0, \ldots, 0) \\
\sum_{j=1}^{N}\left|\alpha_{j}\right| \leq r \Rightarrow\left|\sum_{j=1}^{N} \alpha_{j} \omega_{j}+\varepsilon_{1} \omega_{l_{1}}+\varepsilon_{2} \omega_{l_{2}}\right| \geq \frac{\gamma^{\prime}}{N^{\beta^{\prime}}}
\end{gathered}
$$

Preuve. Il suffit d'appliquer la proposition 5.24 en optimisant en $\varepsilon \in\{0,1,-1\}^{2}$

$$
\begin{gathered}
C(r)=\sum_{\varepsilon} C_{\varepsilon}(r)>0 \quad \beta^{\prime}(r, \gamma)=\max _{\varepsilon} \beta_{\varepsilon}^{\prime}(r, \gamma)>0 \\
\left.\gamma^{\prime}(r)=\min _{\varepsilon} \gamma_{\varepsilon}^{\prime}(r) \in\right] 0, \gamma\left[\quad F_{r, \gamma}=\cap_{\varepsilon} F_{\varepsilon, r, \gamma} \subset \prod_{j}\left[\frac{-1}{2}, \frac{1}{2}\right]\right.
\end{gathered}
$$

En effet

$$
\frac{\gamma_{\varepsilon}^{\prime}}{N^{\beta_{\varepsilon}^{\prime}}} \geq \frac{\gamma^{\prime}}{N^{\beta^{\prime}}} \quad \text { et } \quad \mathbb{P}\left(F_{r, \gamma}^{c}\right)=\mathbb{P}\left(\bigcup_{\varepsilon} F_{\varepsilon, r, \gamma}^{c}\right) \leq \sum_{\varepsilon}\left(1-\mathbb{P}\left(F_{\varepsilon, r, \gamma}\right)\right) \leq \gamma C(r)
$$


La démonstration du théorème 5.20 est achevée en choisissant une unionintersection dénombrable :

$$
F_{k}=\bigcap_{r} \bigcup_{\gamma} F_{r, \gamma}, \quad(r, \gamma) \in(\mathbb{Q} \cap] 0,+\infty[)^{2}
$$

\section{References}

[AG91] S. Alinhac and P. Gérard. Opérateurs pseudo-différentiels et théoreme de Nash-Moser. L'Editeur: EDP Sciences, 1991.

[Bam08] Dario Bambusi. A Birkhoff normal form theorem for some semilinear pdes. NATO Sci. Peace Secur. Ser. B Phys. Biophys., Springer, Dordrecht, pages 213-247, 2008.

[BDGS07] Bambusi, Delort, Grébert, and Szeftel. Almost global existence for Hamiltonian semilinear Klein-Gordon equations with small Cauchy data on Zoll manifolds. Comm. Pure Appl. Math, 60 no 11:pages 1665-1690, 2007.

[Bes78] A.L. Besse. Manifolds all of whose geodesics are closed. Birkhauser, 1978.

[BG04] Bambusi and Grébert. Forme normale pour NLS en dimension quelconque. Compt. Rendu. Acad. Sciences Paris, 2004.

[BG06] D. Bambusi and B. Grébert. Birkhoff normal form for PDEs with tame modulus. Duke Math. J., 135:507-567, 2006.

[BS91] F.A. Berezin and M.A. Shubin. The Schrödinger Equation, volume 66. Springer, 1991.

[BTT10] N. Burq, L. Thomann, and N. Tzvetkov. Long time dynamics for the one dimensional non linear Schrödinger equation. Arxiv preprint arXiv:1002.4054, 2010.

[Che03] D Chelkak. Approximation in the space of spectral data of a perturbed harmonic oscillator. Journal of Mathematical Sciences, 117(3):4260-4269, 2003.

[CKK04] D. Chelkak, P. Kargaev, and E. Korotyaev. Inverse problem for harmonic oscillator perturbed by potential, characterization. Communications in mathematical physics, 249(1):133-196, 2004.

[DS04] J. M. Delort and J. Szeftel. Long-time existence for small data nonlinear Klein-Gordon equations on tori and spheres. Internat. Math. Res. Notices, 37:1897-1966, 2004.

[DS06a] J. Delort and J. Szeftel. Bounded almost global solutions for non hamiltonian semilinear Klein-Gordon equations with radial data on compact revolution hypersurfaces. Annales de l'institut Fourier, 56(5):1419-1456, 2006.

[DS06b] J. M. Delort and J. Szeftel. Long-time existence for semi-linear Klein-Gordon equations with small cauchy data on Zoll manifolds. Amer. J. Math, 128:1187-1218, 2006.

[FG10] Erwan Faou and Benoît Grébert. Quasi-invariant modified Sobolev norms for semi linear reversible pdes. Nonlinearity, 23:pages 429-443, 2010.

[GIP09] Benoit Grébert, Rafik Imekraz, and Eric Paturel. Normal forms for semilinear quantum harmonic oscillators. Comm. Math. Phys., vol 291-3:pages 763-798, 2009.

[GT11] B. Grébert and L. Thomann. Kam for the quantum harmonic oscillator. Comm. Math. Phys., 307-2:383-427, 2011.

[Hel84] Bernard Helffer. Théorie spectrale pour des opérateurs globalement elliptiques, volume 112 of Astérisque. Société Mathématique de France, Paris, 1984. With an English summary.

[Ime12] Rafik Imekraz. Nomal forms for semilinear superquadratic oscillators. Journal of differential equations, vol 252-3:pages 2025-2052, 2012.

[KKP05] M. Klein, E. Korotyaev, and A. Pokrovski. Spectral asymptotics of the harmonic oscillator perturbed by bounded potentials. Annales Henri Poincare, 6(4):747-789, 2005.

[KT05] Herbert Koch and Daniel Tataru. $L^{p}$ eigenfunction bounds for the Hermite operator. Duke Math. J., 128(2):369-392, 2005.

[MT82] H.P. McKean and E. Trubowitz. The spectral class of the quantum-mechanical harmonic oscillator. Communications in Mathematical Physics, 82(4):471-495, 1982.

[Nac69] Leopoldo Nachbin. Topology on spaces of holomorphic mappings. New York, SpringerVerlag, 1969.

[RS75] M. Reed and B. Simon. Methods of modern mathematical physics, vol IV, volume 2. Academic Press, New York - London, 1975. 
[Sze75] Gabor Szego. Orthogonal polynomials. American Mathematical Society, Providence, R.I., fourth edition, 1975. American Mathematical Society, Colloquium Publications, Vol. XXIII.

[Wan09] Wei-Min Wang. Integrals of products of Hermite functions. arXiv:0901.3970, 2009.

Present adress: Laboratoire de Mathématiques Analyse Géométrie Modélisation, Université de Cergy-Pontoise, Site de Saint Martin, 2 avenue Adolphe Chauvin 95302 Cergy-Pontoise Cedex France. Laboratoire Ceremade UMr CNRS 7534, Université Paris-Dauphine, Place du Maréchal De lattre De Tassigny, 75775 Paris CedeX 16 FRANCE

E-mail address: rafik.imekraz@univ-nantes.fr

$U R L:$ http://perso.crans.org/imekraz 